\title{
Effective Lagrangian description of Higgs mediated flavor violating electromagnetic transitions: implications on lepton flavor violation
}

\author{
J. I. Aranda ${ }^{(a)}$, A. Flores-Tlalpa ${ }^{(b)}$, F. Ramírez-Zavaleta ${ }^{(b)}$, F. J. Tlachino ${ }^{(b)}$, J. J. Toscano ${ }^{(b, c)}$, E. S. Tututi ${ }^{(a)}$ \\ (a) Facultad de Ciencias Físico Matemáticas, Universidad Michoacana de San Nicolás de Hidalgo, \\ Avenida Francisco J. Mújica S/N, 58060, Morelia, Michoacán, México. \\ ${ }^{(b)}$ Facultad de Ciencias Físico Matemáticas, Benemérita Universidad \\ Autónoma de Puebla, Apartado Postal 1152, Puebla, Puebla, México. \\ ${ }^{(c)}$ Instituto de Física y Matemáticas, Universidad Michoacana de San Nicolás de Hidalgo, \\ Edificio C-3, Ciudad Universitaria, C.P. 58040, Morelia, Michoacán, México.
}

\begin{abstract}
Higgs mediated flavor violating electromagnetic interactions, induced at the one-loop level by a nondiagonal $H f_{i} f_{j}$ vertex, with $f_{i}$ and $f_{j}$ charged leptons or quarks, are studied within the context of a completely general effective Yukawa sector that comprises $S U_{L}(2) \times U_{Y}(1)$-invariant operators of up to dimension-six. Exact formulae for the one-loop $\gamma f_{i} f_{j}$ and $\gamma \gamma f_{i} f_{j}$ couplings are presented and their related processes used to study the phenomena of Higgs mediated lepton flavor violation. The experimental limit on the $\mu \rightarrow e \gamma$ decay is used to derive a bound on the branching ratio of the $\mu \rightarrow e \gamma \gamma$ transition, which is 6 orders of magnitude stronger than the current experimental limit. Previous results on the $\tau \rightarrow \mu \gamma$ and $\tau \rightarrow \mu \gamma \gamma$ decays are reproduced. The possibility of detecting signals of lepton flavor violation at $\gamma \gamma$ colliders is explored through the $\gamma \gamma \rightarrow l_{i} l_{j}$ reaction, putting special emphasis on the $\tau \mu$ final state. Using the bound imposed on the $H \tau \mu$ vertex by the current experimental data on the muon anomalous magnetic moment, it is found that about half a hundred events may be produced in the International Linear Collider.
\end{abstract}

PACS numbers: 13.35.Dx, 12.60.Fr, 23.20.-g

\section{INTRODUCTION}

The International Linear Collider (ILC) [1] is an ambitious program of electron-positron collisions at the TeV scale, which will provide a clean environment to make studies beyond the capabilities of the Large Hadron Collider(LHC). In addition to complementing and adding precision to the LHC discoveries, the ILC will provide important information to our knowledge of the standard model (SM) and promises access to new physics that could eventually show up at the $\mathrm{TeV}$ scale. In particular, the operation of this collider in the $\gamma \gamma$ mode offers a unique opportunity to explore new physics effects through production mechanisms that are not accessible in leptonic or hadronic machines. This collider will reveal crucial information on those production mechanisms that are naturally suppressed in electron-positron collisions, as the involved cross sections can be significantly larger than the corresponding $e^{+} e^{-}$ones. This is the case of processes that lead to flavor violation, which are quite suppressed within the standard model. In the quark sector of the SM, flavor changing neutral current transitions are very suppressed [2, 3], whereas the analogous transitions in the lepton sector are absent at any order of perturbation theory. Although absent in the SM, the phenomena of lepton flavor violation (LFV) can arise in many of its well-motivated extensions. One new and interesting ingredient of most models beyond the SM is the presence of more complicated Yukawa sectors, which naturally favor nondiagonal interactions mediated by the physical Higgs bosons of the theory. Thus, extended Yukawa sectors are a good place to search for LFV transitions. In particular, one interesting production mechanism would be the one leading to the LFV $\tau \mu$ final state. However, if this transition is mediated by a Higgs boson, one expects an uninteresting cross section for the $e^{+} e^{-} \rightarrow \tau \mu$ production mechanism at the tree level and beyond. The reason is that these types of processes that involve couplings among Higgs bosons and leptons are naturally scaled by the masses of the leptons. In this way, the cross section for the $e^{+} e^{-} \rightarrow \tau \mu$ process is necessarily small, as it is proportional to the product of the three leptonic masses. Although first generated at the one-loop level, the $\gamma \gamma \rightarrow \tau \mu$ mechanism offers a more interesting scenario. One advantage consists in the fact that the cross section depends only of the muon and tau masses, which are substantially larger than the electron mass. In addition, as already mentioned, in many cases the $\gamma \gamma$ cross sections can be much more larger than those associated with $e^{+} e^{-}$collisions.

In this paper we are interested in investigating Higgs mediated flavor violating electromagnetic transitions through the one-loop induced $\gamma f_{i} f_{j}$ and $\gamma \gamma f_{i} f_{j}$ couplings and their related processes, namely, the $f_{i} \rightarrow f_{j} \gamma$ and $f_{i} \rightarrow f_{j} \gamma \gamma$ decays, as well as the $\gamma \gamma \rightarrow f_{i} f_{j}$ reaction in the context of $\gamma \gamma$ colliders. Although we will present exact expressions for these processes, valid for quarks of leptons, we will focus on their implications on LFV. Although, as already mentioned, these types of processes are absent at any order of perturbation theory within the SM, they can be induced in many of its well-motivated extensions. In particular, we will exploit the potential of the $\gamma \gamma \rightarrow \tau \mu$ reaction at $\gamma \gamma$ colliders to search signals of LFV. We will focus on extended Yukawa sectors that are always present within the SM with additional $S U_{L}(2)$-Higgs multiplets or in larger gauge groups. Some processes naturally associated to flavor 
violation could be significantly impacted by Yukawa sectors associated with multi-Higgs models, as it is expected that more complicated Higgs sectors tend to favor this class of new physics effects. We will assume that the $\gamma f_{i} f_{j}$ and $\gamma \gamma f_{i} f_{j}$ couplings are generated by a virtual scalar field with a mass of the order of the Fermi scale $v \approx 246 \mathrm{GeV}$ through the flavor violating $H f_{i} f_{j}$ vertex. However, instead of tackling the problem in a specific model, we will adopt a model independent approach by using the effective Lagrangian technique [4], which is an appropriate scheme to study those processes that are suppressed or forbid in the SM. As it has been shown in Refs. [5, 6], it is not necessary to introduce new degrees of freedom in order to generate flavor violation at the level of classical action; the introduction of operators of dimension higher than four will be enough. We will see below that an effective Yukawa sector that incorporates $S U_{L}(2) \times U_{Y}(1)$-invariants of up to dimension-six is enough to reproduce, in a model-independent manner, the main features that are common to extended Yukawa sectors, such as the presence of both flavor violation and CP violation. Although theories beyond the SM require more complicated Higgs sectors that include new physical scalars, we stress that our approach for studying flavor violating electromagnetic couplings induced by a relatively light scalar particle is sufficiently general to incorporate the most relevant aspects of extended theories, as in most cases, it is always possible to identify in an appropriate limit a SM-like Higgs boson whose couplings to pairs of $W$ and $Z$ bosons coincide with those given in the minimal SM. Besides its model independence, our framework has the advantage that it involves an equal or even smaller number of unknown parameters than those usually appearing in specific extended Yukawa sectors. As already mentioned, one important goal of this work is to calculate the amplitudes for the $\gamma f_{i} f_{j}$ and $\gamma \gamma f_{i} f_{j}$ interactions, which are generated at the one-loop level by the flavor violating $H f_{i} f_{j}$ vertex. We will present exact formulae, which means that the masses of all particles will be conserved, no approximations will be made. These general expressions can be used in searching signals of flavor violation both in the quark sector and in the lepton sector. In a previous paper by some of us [6], the implications of Higgs mediated LFV on the tau decays $\tau \rightarrow \mu \gamma$ and $\tau \rightarrow \mu \gamma \gamma$ were investigated within the context of this model-independent description of extended Higgs sectors. In the present paper, we will concentrate in investigating the potential of extended Yukawa sectors to induce lepton flavor violation via the electromagnetic $\mu \rightarrow e \gamma$ and $\mu \rightarrow e \gamma \gamma$ transitions or through the $\gamma \gamma \rightarrow \tau \mu$ reaction, which is the most promising one to detect possible signals of LFV at the ILC.

The paper has been organized as follows. In Sec. [II, an effective Lagrangian for the Yukawa sector that induces the $H f_{i} f_{j}$ vertex is presented. Section $\amalg$ in devoted to calculating the exact amplitudes for the $\gamma f_{i} f_{j}$ and $\gamma \gamma f_{i} f_{j}$ couplings. In Sec. IV the branching ratios for the $l_{i} \rightarrow l_{j} \gamma$ and $l_{i} \rightarrow l_{j} \gamma \gamma$ decays are studied. In particular, the experimental limit on the $\mu \rightarrow e \gamma$ decays will be used to impose a bound on the $\mu \rightarrow e \gamma \gamma$ transition. Section $\square$ is devoted to calculating and discussing the cross section for the $\gamma \gamma \rightarrow \tau \mu$ reaction in the context of the ILC. Finally, in Sec. VI the conclusions are presented.

\section{EFFECTIVE LAGRANGIAN DESCRIPTION OF HIGGS MEDIATED FLAVOR VIOLATION}

In the SM the Yukawa sector is both flavor- and $\mathrm{CP}$-conserving, but these effects can be generated at the tree level if new scalar fields are introduced. One alternative, which does not contemplate the explicit introduction of new degrees of freedom, consists in incorporating into the classical action the virtual effects of the heavy degrees by introducing $S U_{L}(2) \times U_{Y}(1)$-invariant operators of dimension higher than four [5, 6]. Indeed, it is only necessary to extend the Yukawa sector with dimension-six operators to induce the most general coupling of the Higgs boson to quarks and leptons. A Yukawa sector with these features has the following structure [5, 6 ]:

$$
\begin{aligned}
\mathcal{L}_{\text {eff }}^{Y}= & -Y_{i j}^{l}\left(\bar{L}_{i} \Phi l_{j}\right)-\frac{\alpha_{i j}^{l}}{\Lambda^{2}}\left(\Phi^{\dagger} \Phi\right)\left(\bar{L}_{i} \Phi l_{j}\right)+H . c . \\
& -Y_{i j}^{d}\left(\bar{Q}_{i} \Phi d_{j}\right)-\frac{\alpha_{i j}^{d}}{\Lambda^{2}}\left(\Phi^{\dagger} \Phi\right)\left(\bar{Q}_{i} \Phi d_{j}\right)+H . c . \\
& -Y_{i j}^{u}\left(\bar{Q}_{i} \tilde{\Phi} u_{j}\right)-\frac{\alpha_{i j}^{u}}{\Lambda^{2}}\left(\Phi^{\dagger} \Phi\right)\left(\bar{Q}_{i} \tilde{\Phi} u_{j}\right)+H . c .,
\end{aligned}
$$

where $Y_{i j}, L_{i}, Q_{i}, \Phi, l_{i}, d_{i}$, and $u_{i}$ stand for the usual components of the Yukawa matrix, the left-handed lepton doublet, the left-handed quark doublet, the Higgs doublet, the right-handed charged lepton singlet, and the righthanded quark singlets of down and up type, respectively. The $\alpha_{i j}$ numbers are the components of a $3 \times 3$ general matrix, which parametrize the details of the underlying physics, whereas $\Lambda$ is the typical scale of these new physics effects.

After spontaneous symmetry breaking, this extended Yukawa sector can be diagonalized as usual via the unitary matrices $V_{L}^{l, d, u}$ and $V_{R}^{l, d, u}$, which relate gauge states to mass eigenstates. In the unitary gauge, the diagonalized 
Lagrangian can be written as follows:

$$
\begin{aligned}
\mathcal{L}_{\text {eff }}^{Y}= & -\left(1+\frac{g}{2 m_{W}} H\right)\left(\bar{E} M_{l} E+\bar{D} M_{d} D+\bar{U} M_{u} U\right) \\
& -H\left(1+\frac{g}{4 m_{W}} H\left(3+\frac{g}{2 m_{W}} H\right)\right)\left(\bar{E} \Omega^{l} P_{R} E+\bar{D} \Omega^{d} P_{R} D+\bar{U} \Omega^{u} P_{R} U+H . c .\right),
\end{aligned}
$$

where the $M_{a}(a=l, d, u)$ are the diagonal mass matrix, whereas $\bar{E}=(\bar{e}, \bar{\mu}, \bar{\tau}), \bar{D}=(\bar{d}, \bar{s}, \bar{b})$, and $\bar{U}=(\bar{u}, \bar{c}, \bar{t})$ are vectors in the flavor space. In addition, $\Omega^{a}$ are matrices defined in the flavor space through the relation

$$
\Omega^{a}=\frac{1}{\sqrt{2}}\left(\frac{v}{\Lambda}\right)^{2} V_{L}^{a} \alpha^{a} V_{R}^{a \dagger}
$$

To generate Higgs-mediated flavor violation at the level of classical action, it is assumed that neither $Y^{l, d, u}$ nor $\alpha^{l, d, u}$ are diagonalized by the $V_{L, R}^{a}$ rotation matrices, which should only diagonalize the sum $Y^{l, d, u}+\alpha^{l, d, u}$. As a consequence, mass and interactions terms would not be simultaneously diagonalized as occurs in the dimension-four theory. In addition, if $\Omega^{a \dagger} \neq \Omega^{a}$, the Higgs boson couples to fermions through both scalar and pseudoscalar components, which in turn could lead to $\mathrm{CP}$ violation in some processes. As a consequence, the flavor violating coupling $H f_{i} f_{j}$, where $f$ stands for a charged lepton or quark, has the most general renormalizable structure of scalar and pseudoscalar type given by

$$
-i \Gamma_{i j}=-i\left(\omega_{i j} P_{R}+\omega_{i j}^{*} P_{L}\right)
$$

where $\omega_{i j}=\frac{g m_{i}}{2 m_{W}} \delta_{i j}+\Omega_{i j}, P_{R}=\frac{1+\gamma_{5}}{2}$, and $P_{L}=\frac{1-\gamma_{5}}{2}$. This is the vertex that will be used in the next section to calculate the amplitudes for the $\gamma f_{i} f_{j}$ and $\gamma \gamma f_{i} f_{j}$ couplings, which are induced at the one-loop level.

To close this section, let us to emphasize that the above effective Lagrangian describes the most general coupling of renormalizable type of a scalar field to pairs of fermions, which reproduces the main features of most of extended Yukawa sectors, as the most general version of the two-Higgs doublet model (THDM-III) 7] and multi-Higgs models that comprise additional multiplets of $S U_{L}(2) \times U_{Y}(1)$ or scalar representations of larger gauge groups. Our approach also covers more exotic formulations of flavor violation, as the so-called familon models [8] or theories that involve an Abelian flavor symmetry [9]. In this way, our results will be applicable to a wide variety of models that predict scalar-mediated flavor violation.

\section{THE ONE-LOOP AMPLITUDES FOR THE $\gamma \gamma f_{i} f_{j}$ AND $\gamma f_{i} f_{j}$ COUPLINGS}

In this section, we present the amplitudes associated with the on-shell $\gamma \gamma f_{i} f_{j}$ and $\gamma f_{i} f_{j}$ couplings, which can be used to predict the cross sections or decay widths of their related processes. No approximations will be made and exact formulae will be presented. In particular, the amplitude for the $\gamma \gamma f_{i} f_{j}$ coupling will be given in terms of scalar products among the four-vectors involved. From these expressions, the conversion to the kinematical variables associated with the scattering $\gamma \gamma \rightarrow f_{i} f_{j}$ process (Mandelstam's variables) or with the three-body $f_{i} \rightarrow f_{j} \gamma \gamma$ decays (phase space variables) can be easily performed.

\section{A. The $\gamma \gamma f_{i} f_{j}$ coupling}

The contribution of the flavor violating $H f_{i} f_{j}$ vertex to the $\gamma \gamma f_{i} f_{j}$ coupling is given through the box and reducible diagrams shown in Figs. 1 and 2 each leading separately to a finite and gauge-invariant result. It should be noticed that the contribution of the $H f_{i} f_{j}$ vertex occurs at the one-loop level in graphs of Fig. 1, whereas it contributes at the tree-level in the diagrams of Fig. 2. This last contribution, which is determined by the one-loop $H \gamma \gamma$ vertex, can eventually have a determinant role in the cross section due to an resonant effect of the Higgs boson. In the case of those processes involving light fermions, such as the LFV $\gamma \gamma \rightarrow \tau \mu$ reaction, the contributions arising from diagrams of Fig. 1 are marginal due to the presence (at first order in the $\Omega_{\tau \mu}$ parameter) of the SM coupling $H \tau \tau$, which is proportional to $m_{\tau} / v$. However, the contributions given by these diagrams can be competitive with that induced by the diagrams of Fig. 2, as this suppression effect is not present in flavor violating processes involving the quark top [10]. We have adopted the convention of taking all momenta incoming: $k_{1}+k_{2}+p_{i}+p_{j}=0$, where $k_{1,2}$ and $p_{i, j}$ stand for the four-vectors of photons and fermions, respectively. The rest of our notation is indicated in Figs. 11 and 2.

The analytical structure of the amplitude is dictated by electromagnetic gauge invariance and Bose statistics. In addition, since the structure of the $H f_{i} f_{j}$ vertex is of renormalizable type, the amplitude must be free of ultraviolet 
divergences. We first analyze the contribution given by the set of diagrams shown in Fig. 1 The corresponding amplitude can be written as follows:

$$
\mathcal{M}_{\text {Box }+ \text { red }}^{\mu \nu}=e^{2} \overline{\mathrm{v}}_{j}\left(p_{j}\right) \int \frac{d^{D} k}{(2 \pi)^{D}}\left[\sum_{n=1}^{2} \frac{\mathcal{T}_{B_{n}}^{\mu \nu}}{\Delta_{B_{n}}}+\sum_{n=1}^{4} \frac{\mathcal{T}_{T_{n}}^{\mu \nu}}{\Delta_{T_{n}}}+\sum_{n=1}^{6} \frac{\mathcal{T}_{S_{n}}^{\mu \nu}}{\Delta_{S_{n}}}\right] \mathrm{u}_{i}\left(p_{i}\right),
$$

where the subindexes $B, T$, and $S$ stand for box, triangle, and self-energy diagrams, respectively. The diverse magnitudes appearing in these expressions are given by

$$
\begin{aligned}
& \mathcal{T}_{B_{1}}^{\mu \nu}=\left(\omega_{k j} P_{R}+\omega_{k j}^{*} P_{L}\right)\left(\not k-\not p_{j}+m_{k}\right) \gamma^{\nu}\left(\not k+\not k p_{1}+\not p_{i}+m_{k}\right) \gamma^{\mu}\left(\not k+\not p_{i}+m_{k}\right)\left(\omega_{i k} P_{R}+\omega_{i k}^{*} P_{L}\right) \text {, } \\
& \mathcal{T}_{B_{2}}^{\mu \nu}=\left(\omega_{k j} P_{R}+\omega_{k j}^{*} P_{L}\right)\left(\not k-\not k j+m_{k}\right) \gamma^{\mu}\left(\not k+\not k k_{2}+\not p_{i}+m_{k}\right) \gamma^{\nu}\left(\not k+\not k+m_{k}\right)\left(\omega_{i k} P_{R}+\omega_{i k}^{*} P_{L}\right) \text {, } \\
& \mathcal{T}_{T_{1}}^{\mu \nu}=\left(\omega_{k j} P_{R}+\omega_{k j}^{*} P_{L}\right)\left(\not k-\not p_{j}+m_{k}\right) \gamma^{\nu}\left(\not k+\not k k_{1}+\not p_{i}+m_{k}\right)\left(\omega_{i k} P_{R}+\omega_{i k}^{*} P_{L}\right)\left(\not k_{1}+\not p_{i}+m_{i}\right) \gamma^{\mu} \text {, } \\
& \mathcal{T}_{T_{2}}^{\mu \nu}=\left(\omega_{k j} P_{R}+\omega_{k j}^{*} P_{L}\right)\left(\not k-\not p_{j}+m_{k}\right) \gamma^{\mu}\left(\not k+\not k k_{2}+\not p_{i}+m_{k}\right)\left(\omega_{i k} P_{R}+\omega_{i k}^{*} P_{L}\right)\left(\not k k_{2}+\not p_{i}+m_{i}\right) \gamma^{\nu} \text {, } \\
& \mathcal{T}_{T_{3}}^{\mu \nu}=\gamma^{\nu}\left(\not k_{1}+\not p_{i}+m_{j}\right)\left(\omega_{k j} P_{R}+\omega_{k j}^{*} P_{L}\right)\left(\not k+\not k k_{1}+\not p_{i}+m_{k}\right) \gamma^{\mu}\left(\not k+\not k i+m_{k}\right)\left(\omega_{i k} P_{R}+\omega_{i k}^{*} P_{L}\right) \text {, } \\
& \mathcal{T}_{T_{4}}^{\mu \nu}=\gamma^{\mu}\left(\not k_{2}+\not p_{i}+m_{j}\right)\left(\omega_{k j} P_{R}+\omega_{k j}^{*} P_{L}\right)\left(\not k+\not k k_{2}+\not p_{i}+m_{k}\right) \gamma^{\nu}\left(\not k+\not k i+m_{k}\right)\left(\omega_{i k} P_{R}+\omega_{i k}^{*} P_{L}\right) \text {, } \\
& \mathcal{T}_{S_{1}}^{\mu \nu}=\gamma^{\mu}\left(-\not p_{j}-\not k_{1}+m_{j}\right) \gamma^{\nu}\left(\not p_{i}+m_{i}\right)\left(\omega_{k j} P_{R}+\omega_{k j}^{*} P_{L}\right)\left(\not k+\not p_{i}+m_{k}\right)\left(\omega_{i k} P_{R}+\omega_{i k}^{*} P_{L}\right) \text {, } \\
& \mathcal{T}_{S_{2}}^{\mu \nu}=\gamma^{\nu}\left(-\not p_{j}-\not k_{2}+m_{j}\right) \gamma^{\mu}\left(\not p_{i}+m_{i}\right)\left(\omega_{k j} P_{R}+\omega_{k j}^{*} P_{L}\right)\left(\not k+\not k i+m_{k}\right)\left(\omega_{i k} P_{R}+\omega_{i k}^{*} P_{L}\right) \text {, } \\
& \mathcal{T}_{S_{3}}^{\mu \nu}=\gamma^{\mu}\left(-\not p_{j}-\not k_{1}+m_{j}\right)\left(\omega_{k j} P_{R}+\omega_{k j}^{*} P_{L}\right)\left(\not k-\not k_{1}-\not p_{j}+m_{k}\right)\left(\omega_{i k} P_{R}+\omega_{i k}^{*} P_{L}\right)\left(-\not k_{1}-\not p_{j}+m_{i}\right) \gamma^{\nu} \text {, } \\
& \mathcal{T}_{S_{4}}^{\mu \nu}=\gamma^{\nu}\left(-\not p_{j}-\not k_{2}+m_{j}\right)\left(\omega_{k j} P_{R}+\omega_{k j}^{*} P_{L}\right)\left(\not k-\not k k_{2}-\not p_{j}+m_{k}\right)\left(\omega_{i k} P_{R}+\omega_{i k}^{*} P_{L}\right)\left(-\not k_{2}-\not p_{j}+m_{i}\right) \gamma^{\mu} \text {, } \\
& \mathcal{T}_{S_{5}}^{\mu \nu}=\left(\omega_{k j} P_{R}+\omega_{k j}^{*} P_{L}\right)\left(\not k-\not p_{j}+m_{k}\right)\left(\omega_{i k} P_{R}+\omega_{i k}^{*} P_{L}\right)\left(-\not p_{j}+m_{i}\right) \gamma^{\nu}\left(\not k_{1}+\not p_{i}+m_{i}\right) \gamma^{\mu} \text {, } \\
& \mathcal{T}_{S_{6}}^{\mu \nu}=\left(\omega_{k j} P_{R}+\omega_{k j}^{*} P_{L}\right)\left(\not k-\not p_{j}+m_{k}\right)\left(\omega_{i k} P_{R}+\omega_{i k}^{*} P_{L}\right)\left(-\not p_{j}+m_{i}\right) \gamma^{\mu}\left(\not k_{2}+\not p_{i}+m_{i}\right) \gamma^{\nu} \text {, }
\end{aligned}
$$

and

$$
\begin{aligned}
& \Delta_{B_{n}}=\left[\left(k-p_{j}\right)^{2}-m_{k}^{2}\right]\left[\left(k+k_{n}+p_{i}\right)^{2}-m_{k}^{2}\right]\left[\left(k+p_{i}\right)^{2}-m_{k}^{2}\right]\left[k^{2}-m_{H}^{2}\right], \\
& \Delta_{T_{n}}=\left\{\begin{array}{ll}
{\left[\left(k-p_{j}\right)^{2}-m_{k}^{2}\right]\left[\left(k+k_{n}+p_{i}\right)^{2}-m_{k}^{2}\right]\left[\left(k_{n}+p_{i}\right)^{2}-m_{i}^{2}\right]\left[k^{2}-m_{H}^{2}\right],} & n=1,2 \\
{\left[\left(k_{n-2}+p_{i}\right)^{2}-m_{i}^{2}\right]\left[\left(k+k_{n-2}+p_{i}\right)^{2}-m_{k}^{2}\right]\left[\left(k+p_{i}\right)^{2}-m_{k}^{2}\right]\left[k^{2}-m_{H}^{2}\right],} & n=3,4
\end{array},\right. \\
& \Delta_{S_{n}}=\left\{\begin{array}{ll}
{\left[\left(p_{j}+k_{n}\right)^{2}-m_{j}^{2}\right]\left[p_{i}^{2}-m_{i}^{2}\right]\left[\left(k+p_{i}\right)^{2}-m_{k}^{2}\right]\left[k^{2}-m_{H}^{2}\right],} & n=1,2 \\
{\left[\left(p_{j}+k_{n-2}\right)^{2}-m_{j}^{2}\right]\left[\left(k-p_{j}-k_{n-2}\right)^{2}-m_{k}^{2}\right]\left[\left(p_{j}+k_{n-2}\right)^{2}-m_{i}^{2}\right]\left[k^{2}-m_{H}^{2}\right],} & n=3,4 \\
{\left[\left(k-p_{j}\right)^{2}-m_{k}^{2}\right]\left[p_{j}^{2}-m_{i}^{2}\right]\left[\left(k_{n-4}+p_{i}\right)^{2}-m_{i}^{2}\right]\left[k^{2}-m_{H}^{2}\right],} & n=5,6
\end{array} .\right.
\end{aligned}
$$

Gauge invariance means that the amplitude must satisfy the transversality conditions,

$$
\begin{aligned}
& k_{1 \mu} \mathcal{M}_{\text {Box +red }}^{\mu \nu}\left(k_{1}, k_{2}\right)=0, \\
& k_{2 \nu} \mathcal{M}_{\text {Box +red }}^{\mu \nu}\left(k_{1}, k_{2}\right)=0,
\end{aligned}
$$

whereas Bose statics requires a symmetric amplitude under the interchanges $k_{1} \leftrightarrow k_{2}$ and $\mu \leftrightarrow \nu$ :

$$
\mathcal{M}_{\text {Box +red }}^{\mu \nu}\left(k_{1}, k_{2}\right)=\mathcal{M}_{\text {Box+red }}^{\nu \mu}\left(k_{2}, k_{1}\right) \text {. }
$$

To solve the above integrals, we have used the Passarino-Veltman Lorentz tensorial decomposition [11] implemented in the FeynCalc computer program [12]. Although, in order to make less cumbersome the expressions, we present our results in terms of the form factors that define this Lorentz decomposition, we have verified that they are free of ultraviolet divergences by performing the reduction of these form factors to $B_{0}, C_{0}$, and $D_{0}$ scalar functions. Although the box diagrams depend on $B_{0}$ functions, the divergences cancel among themselves dealing to a finite amplitude. This is in contrast with the sets of triangle and self-energy graphs, which generate divergent pieces that disappear only after adding both sets of amplitudes. This cancellation of ultraviolet divergences is quite intricate due to the fact that the amplitudes depend on the $B_{0}$ functions in a very complicated way. It is worth mentioning that a similar problem is present in the amplitude for the one-loop $t \rightarrow c g g$ decay of the quark top within the context of the SM. The amplitude that presents this problem is induced by the contribution of the pseudo-Goldstone boson associated with the $W$ gauge boson, as it was studied in Ref. [3]. The authors of this reference verified numerically that their 
results are free of divergences. In our case, we carried out an analytical analysis in order to convince ourselves that these divergences are absent indeed. To perform this analysis, we replace each of the $B_{0}$ functions by its divergent part, which is common to all them, namely, we put $B_{0} \rightarrow \Delta=-1 /(D-4)$ in our amplitude in order to express the apparently divergent part in a product of the way $F R^{\mu \nu} \Delta$, with $F$ a scalar function that depends in complicated way on kinematics variables and $R^{\mu \nu}$ a nontrivial Lorentz tensor structure. After some nontrivial algebraic manipulations, we achieve the required result $F=0$, which shows that in fact the amplitude is free of ultraviolet divergences. As to gauge invariance, it is achieved only after adding the amplitudes of all diagrams of Fig. 1, On the other hand, the contribution from the set of diagrams shown in Fig. 2] is free of ultraviolet divergences and is gauge-invariant, as it is generated essentially by the SM one-loop $H \gamma \gamma$ vertex, which, as is well-known [13], is characterized by a finite and gauge-invariant amplitude. After these considerations, the complete amplitude for the $\gamma \gamma f_{i} f_{j}$ coupling can be conveniently written in the following way:

$$
\mathcal{M}^{\mu \nu}=\frac{\alpha}{4 \pi} \overline{\mathrm{v}}_{j}\left(p_{j}\right)\left[\frac{1}{m_{W}} \Gamma_{\mathrm{Box}+\mathrm{red}}^{\mu \nu}+\frac{g}{2 m_{W}} \Gamma_{j i} \Gamma_{\mathrm{red}(\mathrm{H})}^{\mu \nu}\right] \mathrm{u}_{i}\left(p_{i}\right)
$$

where $\Gamma_{\mathrm{Box}+\mathrm{red}}^{\mu \nu}$ and $\Gamma_{\mathrm{red}(\mathrm{H})}^{\mu \nu}$ represent the contributions coming from the sets of diagrams given in Figs. 1 and 2 , respectively. We have normalized the amplitudes to the $W$ gauge boson mass $m_{W}$. The $\Gamma_{\text {Box }+ \text { red }}^{\mu \nu}$ tensor amplitude can be organized in terms of 11 gauge structures as follows:

$$
\Gamma_{\mathrm{Box}+\mathrm{red}}^{\mu \nu}=\sum_{i=1}^{11}\left(T_{i_{R}}^{\mu \nu} P_{R}+T_{i_{L}}^{\mu \nu} P_{L}\right),
$$

where gauge structures means that they satisfy the transversality conditions

$$
\begin{aligned}
& k_{1 \mu} T_{i_{R, L}}^{\mu \nu}=0, \\
& k_{2 \nu} T_{i_{R, L}}^{\mu \nu}=0 .
\end{aligned}
$$

We have arranged our results in an order that reflects in a manifest way both gauge invariance and Bose symmetry. Accordingly, each of the $T_{i_{R, L}}^{\mu \nu}$ quantities are made of products that contain a gauge structure and a scalar form factor, which respects both symmetries as a whole. These tensor amplitudes are given by:

$$
\begin{aligned}
& T_{1_{L, R}}^{\mu \nu}=F_{1_{L, R}} \frac{g^{\mu \nu} k_{1} \cdot k_{2}-k_{1}^{\nu} k_{2}^{\mu}}{k_{1} \cdot k_{2}}, \\
& T_{2_{L, R}}^{\mu \nu}=F_{2_{L, R}} \frac{\left(p_{j}^{\mu} k_{1} \cdot k_{2}-k_{2}^{\mu} k_{1} \cdot p_{j}\right)\left(p_{j}^{\nu} k_{1} \cdot k_{2}-k_{1}^{\nu} k_{2} \cdot p_{j}\right)}{\left(m_{W} k_{1} \cdot k_{2}\right)^{2}}, \\
& T_{3_{L, R}}^{\mu \nu}=\left(F_{3_{L, R}} \not k_{1}+F_{4_{L, R}} \not k_{2}\right) \frac{\left(g^{\mu \nu} k_{1} \cdot k_{2}-k_{1}^{\nu} k_{2}^{\mu}\right)}{m_{W} k_{1} \cdot k_{2}}, \\
& T_{4_{L, R}}^{\mu \nu}=F_{5_{L, R}} \frac{\left(\gamma^{\mu} k_{1} \cdot k_{2}-k_{2}^{\mu} \not k_{1}\right)\left(p_{j}^{\nu} k_{1} \cdot k_{2}-k_{1}^{\nu} k_{2} \cdot p_{j}\right)}{m_{W}\left(k_{1} \cdot k_{2}\right)^{2}}+F_{6_{L, R}} \frac{\left(\gamma^{\nu} k_{1} \cdot k_{2}-k_{1}^{\nu} \not k_{2}\right)\left(p_{j}^{\mu} k_{1} \cdot k_{2}-k_{2}^{\mu} k_{1} \cdot p_{j}\right)}{m_{W}\left(k_{1} \cdot k_{2}\right)^{2}} \text {, } \\
& T_{5_{L, R}}^{\mu \nu}=\left(F_{7_{L, R}} \not k_{1}+F_{8_{L, R}} \not k_{2}\right) \frac{\left(p_{j}^{\mu} k_{1} \cdot k_{2}-k_{2}^{\mu} k_{1} \cdot p_{j}\right)\left(p_{j}^{\nu} k_{1} \cdot k_{2}-k_{1}^{\nu} k_{2} \cdot p_{j}\right)}{m_{W}^{3}\left(k_{1} \cdot k_{2}\right)^{2}}, \\
& T_{6_{L, R}}^{\mu \nu}=F_{9_{L, R}} \frac{\not k_{1} \gamma^{\mu}\left(p_{j}^{\nu} k_{1} \cdot k_{2}-k_{1}^{\nu} k_{2} \cdot p_{j}\right)}{m_{W}^{2} k_{1} \cdot k_{2}}+F_{10_{L, R}} \frac{\not k_{2} \gamma^{\nu}\left(p_{j}^{\mu} k_{1} \cdot k_{2}-k_{2}^{\mu} k_{1} \cdot p_{j}\right)}{m_{W}^{2} k_{1} \cdot k_{2}}, \\
& T_{7_{L, R}}^{\mu \nu}=F_{11_{L, R}} \frac{\gamma^{\mu} \not k_{2} \gamma^{\nu} k_{1} \cdot k_{2}-\not k_{1} \not k_{2} \gamma^{\nu} k_{2}^{\mu}}{m_{W} k_{1} \cdot k_{2}}+F_{12_{L, R}} \frac{\gamma^{\nu} \not k_{1} \gamma^{\mu} k_{1} \cdot k_{2}-\not k_{2} \not k_{1} \gamma^{\mu} k_{1}^{\nu}}{m_{W} k_{1} \cdot k_{2}} \text {, } \\
& T_{8_{L, R}}^{\mu \nu}=F_{13_{L, R}} \frac{\not k_{1} \gamma^{\mu} \gamma^{\nu} k_{1} \cdot k_{2}-\not k_{1} \gamma^{\mu} \not k_{2} k_{1}^{\nu}}{m_{W} k_{1} \cdot k_{2}}+F_{14_{L, R}} \frac{\not k_{2} \gamma^{\nu} \gamma^{\mu} k_{1} \cdot k_{2}-\not k_{2} \gamma^{\nu} \not k_{1} k_{2}^{\mu}}{m_{W} k_{1} \cdot k_{2}}, \\
& T_{9_{L, R}}^{\mu \nu}=F_{15_{L, R}} \frac{\not k_{1} \not k_{2} \gamma^{\nu}\left(p_{j}^{\mu} k_{1} \cdot k_{2}-k_{2}^{\mu} k_{1} \cdot p_{j}\right)}{m_{W}^{3} k_{1} \cdot k_{2}}+F_{16_{L, R}} \frac{\not k_{2} \not k_{1} \gamma^{\mu}\left(p_{j}^{\nu} k_{1} \cdot k_{2}-k_{1}^{\nu} k_{2} \cdot p_{j}\right)}{m_{W}^{3} k_{1} \cdot k_{2}} \text {, } \\
& T_{10_{L, R}}^{\mu \nu}=F_{17_{L, R}} \frac{\not k_{1} \gamma^{\mu} \not k_{2}\left(p_{j}^{\nu} k_{1} \cdot k_{2}-k_{1}^{\nu} k_{2} \cdot p_{j}\right)}{m_{W}^{3} k_{1} \cdot k_{2}}+F_{18_{L, R}} \frac{\not k_{2} \gamma^{\nu} \not k_{1}\left(p_{j}^{\mu} k_{1} \cdot k_{2}-k_{2}^{\mu} k_{1} \cdot p_{j}\right)}{m_{W}^{3} k_{1} \cdot k_{2}} \text {, } \\
& T_{11_{L, R}}^{\mu \nu}=F_{19_{L, R}} \frac{\not k_{1} \gamma^{\mu} \not k_{2} \gamma^{\nu}}{m_{W}^{2}}+F_{20_{L, R}} \frac{\not k_{2} \gamma^{\nu} \not k_{1} \gamma^{\mu}}{m_{W}^{2}}
\end{aligned}
$$




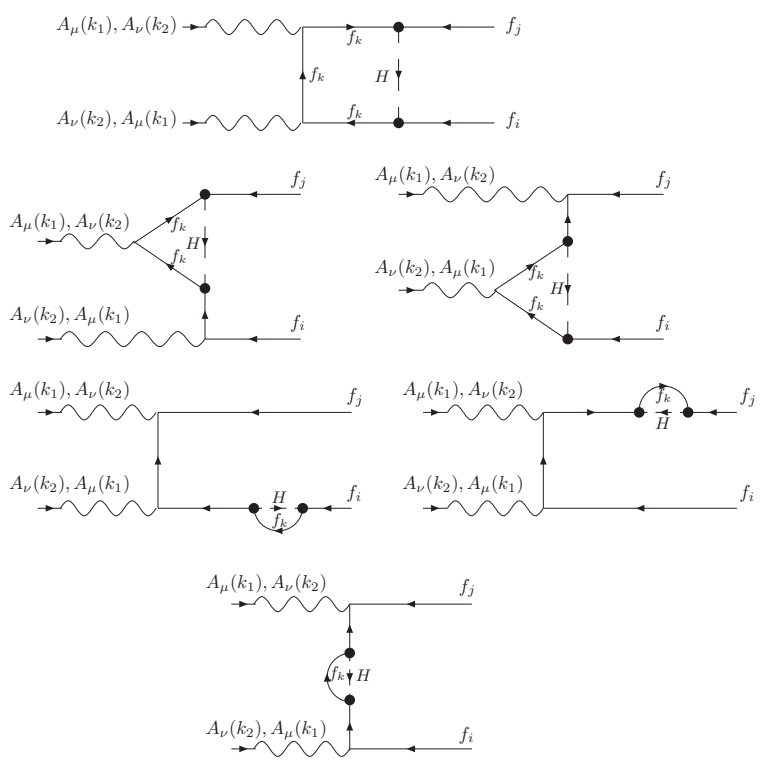

FIG. 1: Box and reducible diagrams contributing to the $\gamma \gamma f_{i} f_{j}$ coupling.

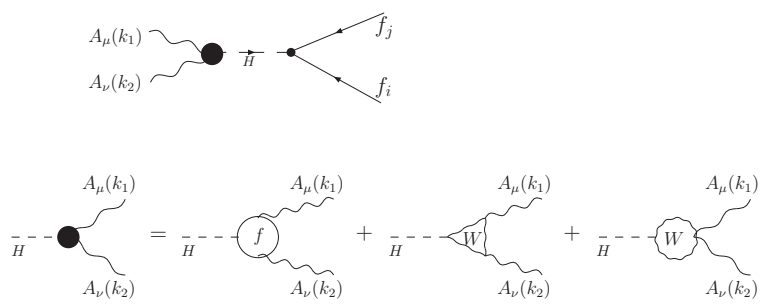

FIG. 2: Contribution of the standard model loop-induced $H \gamma \gamma$ vertex to the $\gamma \gamma f_{i} f_{j}$ coupling.

The fact that each $T_{i_{R, L}}^{\mu \nu}$ term respects the Bose symmetry means that, for example, $F_{19_{R, L}}$ transforms into $F_{20_{R, L}}$ under the interchanges $k_{1} \leftrightarrow k_{2}$ and $\mu \leftrightarrow \nu$, but $F_{1_{R, L}}$ remains invariant under the same interchanges. On the other hand, the $\Gamma_{\text {red(H) }}^{\mu \nu}$ amplitude is given by

$$
\Gamma_{\text {red(H) }}^{\mu \nu}=F_{21} \frac{k_{2}^{\mu} k_{1}^{\nu}-k_{1} \cdot k_{2} g^{\mu \nu}}{2 k_{1} \cdot k_{2}-m_{H}^{2}+i m_{H} \Gamma_{H}} .
$$

The form factors $F_{n_{R, L}}$ are presented in the Appendix.

\section{B. The $\gamma f_{i} f_{j}$ coupling}

The contribution of the $H f_{i} f_{j}$ vertex to the $\gamma f_{i} f_{j}$ coupling is given by the diagrams shown in Fig. 3 The amplitude is entirely governed by electromagnetic gauge invariance. In principle, the amplitude could depend on Lorentz structures of the following type: monopole $\left(\gamma_{\mu}\right)$, anapole $\left(q^{2} \gamma_{\mu}-\not q q_{\mu}\right)$, magnetic dipole $\left(\sigma_{\mu \nu} q^{\nu}\right)$, and electric dipole $\left(\gamma_{5} \sigma_{\mu \nu} q^{\nu}\right)$. However, since this is a flavor violating transition, there is no contribution to the monopole. Also, the anapolar structure is absent in this case because the three particles are on-shell. So, the amplitude must be made of two pieces, one $\mathrm{CP}$-conserving associated with a magnetic dipole of transition and the other being $\mathrm{CP}$-violating, which is associated with an electric dipole of transition. The latter amplitude only can arise if an imaginary part of the $\omega_{i j}$ parameters is assumed. Since the anomalous $H f_{i} f_{j}$ vertex has a renormalizable structure, these amplitudes must be free of ultraviolet divergences by renormalization theory. As in the previous case, we will take all momenta incoming, i.e., $p_{i}+p_{j}+q=0$, with $q$ the photon four-vector. Then, the vertex associated with the $\gamma f_{i} f_{j}$ coupling is given by

$$
\Gamma_{\mu}=e \int \frac{d^{D} k}{(2 \pi)^{D}}\left(\frac{T_{\mu}^{1}}{\Delta_{1}}+\frac{T^{2}}{\Delta_{2}} \frac{-p_{j}+m_{i}}{m_{j}^{2}-m_{i}^{2}} \gamma_{\mu}+\gamma_{\mu} \frac{p_{i}+m_{j}}{m_{i}^{2}-m_{j}^{2}} \frac{T^{3}}{\Delta_{3}}\right),
$$



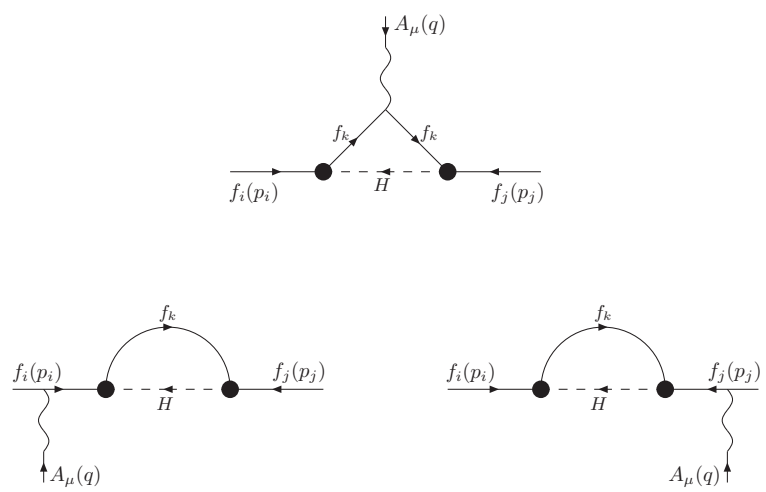

FIG. 3: Diagrams contributing to the $\gamma f_{i} f_{j}$ vertex.

where

$$
\begin{aligned}
T_{\mu}^{1} & =\Gamma_{k j}\left(\not k-\not p_{j}+m_{k}\right) \gamma_{\mu}\left(\not k+\not p_{i}+m_{k}\right) \Gamma_{i k}, \\
T^{2} & =\Gamma_{k j}\left(\not k-\not p_{j}+m_{k}\right) \Gamma_{i k}, \\
T^{3} & =\Gamma_{k j}\left(\not k+\not p_{i}+m_{k}\right) \Gamma_{i k}, \\
\Delta_{1} & =\left[k^{2}-m_{H}^{2}\right]\left[\left(k+p_{i}\right)^{2}-m_{k}^{2}\right]\left[\left(k-p_{j}\right)^{2}-m_{k}^{2}\right], \\
\Delta_{2} & =\left[k^{2}-m_{H}^{2}\right]\left[\left(k-p_{j}\right)^{2}-m_{k}^{2}\right], \\
\Delta_{3} & =\left[k^{2}-m_{H}^{2}\right]\left[\left(k+p_{i}\right)^{2}-m_{k}^{2}\right] .
\end{aligned}
$$

Once the integrals are solved, one can see that the dependence on $\gamma_{\mu}$ cancels exactly after using the Gordon identity, which allows us to eliminate the dependence of the amplitude on the four-vector $p_{i \mu}$. The result can be written in a symmetric form as follows:

$$
\Gamma_{\mu}=\frac{i \alpha}{8 \pi s_{W} m_{W}}\left(\Omega_{i j}^{R} A\left(x_{i}, x_{j}, x_{k}\right) i \sigma_{\mu \nu} q^{\nu}+\Omega_{i j}^{I} \tilde{A}\left(x_{i}, x_{j}, x_{k}\right) \gamma_{5} \sigma_{\mu \nu} q^{\nu}\right)
$$

where

$$
\begin{aligned}
& A\left(x_{i}, x_{j}, x_{k}\right)=\left(x_{i} \delta_{k i}+x_{j} \delta_{k j}\right) F\left(x_{i}, x_{j}, x_{k}\right), \\
& \tilde{A}\left(x_{i}, x_{j}, x_{k}\right)=\left(x_{i} \delta_{k i}+x_{j} \delta_{k j}\right) \tilde{F}\left(x_{i}, x_{j}, x_{k}\right) \text {, } \\
& F\left(x_{i}, x_{j}, x_{k}\right)=\frac{1}{2\left(x_{i}+x_{j}\right)}\left(-1-x_{k}\left(x_{i}+x_{j}+x_{k}\right) 2 m_{H}^{2} C_{0}+\frac{2\left(1-2 x_{k}^{2}\right)-2 x_{k}\left(x_{i}+x_{j}\right)+x_{i} x_{j}}{x_{i}^{2}-x_{j}^{2}}\left(B_{0}(1)-B_{0}(2)\right)+\right. \\
& \left.\frac{1-x_{k}^{2}}{x_{i}^{2}-x_{j}^{2}}\left(\frac{x_{i}}{x_{j}}\left(B_{0}(3)-B_{0}(2)\right)-\frac{x_{j}}{x_{i}}\left(B_{0}(3)-B_{0}(1)\right)\right)\right) \text {, } \\
& \tilde{F}\left(x_{i}, x_{j}, x_{k}\right)=\frac{1}{2\left(x_{i}+x_{j}\right)}\left(-1-x_{k}\left(x_{i}-x_{j}+x_{k}\right) 2 m_{H}^{2} C_{0}+\frac{2\left(1-2 x_{k}^{2}\right)-2 x_{k}\left(x_{i}-x_{j}\right)-x_{i} x_{j}}{x_{i}^{2}-x_{j}^{2}}\left(B_{0}(1)-B_{0}(2)\right)+\right. \\
& \left.\frac{1-x_{k}^{2}}{x_{i}^{2}-x_{j}^{2}}\left(\frac{x_{i}}{x_{j}}\left(B_{0}(2)-B_{0}(3)\right)-\frac{x_{j}}{x_{i}}\left(B_{0}(1)-B_{0}(3)\right)\right)\right) \text {, }
\end{aligned}
$$

In the above expressions, $\Omega_{i j}^{R}=\operatorname{Re}\left(\Omega_{i j}\right), \Omega_{i j}^{I}=\operatorname{Im}\left(\Omega_{i j}\right)$, and $s_{W}=\sin \theta_{W}$. In addition, the dimensionless variables $x_{a}=m_{a} / m_{H}$ were introduced. Also, $C_{0}=C_{0}\left(m_{i}^{2}, m_{j}^{2}, 0, m_{k}^{2}, m_{H}^{2}, m_{k}^{2}\right), B_{0}(3)=B_{0}\left(0, m_{H}^{2}, m_{k}^{2}\right)$, $B_{0}(1)=B_{0}\left(m_{i}^{2}, m_{H}^{2}, m_{k}^{2}\right)$, and $B_{0}(2)=B_{0}\left(m_{j}^{2}, m_{H}^{2}, m_{k}^{2}\right)$ are Passarino-Veltman scalar functions. 


\section{THE $l_{i} \rightarrow l_{j} \gamma$ AND $l_{i} \rightarrow l_{j} \gamma \gamma$ DECAYS}

In this section, we analyze the branching ratios for the $l_{i} \rightarrow l_{j} \gamma$ and $l_{i} \rightarrow l_{j} \gamma \gamma$ decays and confront our prediction with current experimental limits. We will concentrate in the $\mu \rightarrow e \gamma$ and $\mu \rightarrow e \gamma \gamma$ transitions, as those involving the $\tau$ lepton were already studied in Ref. [6], in which the limits $\operatorname{Br}(\tau \rightarrow \mu \gamma)<7.5 \times 10^{-10}$ and $\operatorname{Br}(\tau \rightarrow \mu \gamma \gamma)<2.3 \times 10^{-12}$ were derived.

\section{A. Experimental constraints from $l_{i} \rightarrow l_{j} \gamma$ and $\mu \rightarrow e \bar{e} e$}

From Eqs. (24, 27, 28), it is straightforward to construct the branching ratio for the $f_{i} \rightarrow f_{j} \gamma$ decay. It can be written as:

$$
B r\left(f_{i} \rightarrow f_{j} \gamma\right)=\frac{\alpha^{2}}{2^{9} \pi^{3} s_{W}^{2}}\left(\frac{m_{i}}{\Gamma_{i}}\right)\left(\frac{m_{H}}{m_{W}}\right)^{2} x_{i}^{2}\left(1-\frac{x_{j}^{2}}{x_{i}^{2}}\right)^{3}\left(\left(\Omega_{i j}^{R}\right)^{2}\left|A\left(x_{i}, x_{j}\right)\right|^{2}+\left(\Omega_{i j}^{I}\right)^{2}\left|\tilde{A}\left(x_{i}, x_{j}\right)\right|^{2}\right)
$$

where $\Gamma_{i}$ is the total decay width of the fermion $f_{i}$. This is an exact result, which can be used for studying Higgs mediated flavor violation in both the quark and the lepton sectors, but in this paper we will focus on LFV transitions. In particular, it is interesting to use the current experimental limits on the LFV $l_{i} \rightarrow l_{j} \gamma$ transitions to constrain the $\Omega_{i j}$ parameters. We will focus on the LFV transitions of the muon. It is important to comment at this point that the complex component of $\Omega_{\mu e}$ also contributes to the electric dipole moment $\left(d_{e}\right)$ of the electron. It can be shown that the current limit on $d_{e}$ induces a very strong bound on $\Omega_{\mu e}^{I}[14]$, so from now on this contribution will be neglected.

The limit on the branching ratio of the $\mu \rightarrow e \gamma$ decay reported by the Particle Data Group [15] is

$$
B r_{e x p}(\mu \rightarrow e \gamma)<1.2 \times 10^{-11}
$$

Then, working out at first order in the $\Omega_{\mu e}^{R} \equiv \Omega_{\mu e}$ parameter, one obtains the following constraint

$$
\Omega_{\mu e}<7 \times 10^{-3}
$$

This bound was obtained assuming a value for the Higgs mass of about the lower limit imposed by LEP on the Higgs mass [16]. In the next subsection, we will use this constraint to impose a bound on the $\mu \rightarrow e \gamma \gamma$ decay.

It is interesting to compare this bound with the one that can be derived from the experimental limit on the branching ratio of the tree-body decay $\mu \rightarrow e e e$, which is strongly restricted by the experiment. In the context of our effective theory, this decay can occur through a virtual Higgs boson as it is shown in Fig. 4. The amplitude is governed by the LFV $H \mu$ e vertex and the SM one Hee, which is very suppressed. The corresponding branching ratio can be written as follows:

$$
B r(\mu \rightarrow e e e)=\frac{\alpha|\Omega \mu e|^{2}}{256 \pi^{2} s_{W}^{2}}\left(\frac{m_{e}}{m_{W}}\right)^{2}\left(\frac{m_{\mu}}{\Gamma_{\mu}}\right) f(y, z)
$$

where

$$
f(y, z)=\int_{0}^{1} d x \frac{z(1-x)^{2}}{(x-y)^{2}+y z}
$$

In the above expressions, $y=m_{H}^{2} / m_{\mu}^{2}$ and $z=\Gamma_{H}^{2} / m_{\mu}^{2}$, where $\Gamma_{\mu}$ and $\Gamma_{H}$ are the muon and Higgs total decay widths, respectively. To bound the $\Omega_{\mu e}$ parameter, we use the limit for $\operatorname{Br}(\mu \rightarrow$ eee $)$ reported by the Particle Data Group [15], which is given by

$$
B r_{E x p}(\mu \rightarrow e e e)<10^{-12}
$$

In Fig. 5 the behavior of $\left|\Omega_{\mu e}\right|^{2}$ as a function the Higgs mass is displayed. It can be appreciated from this figure that $\left|\Omega_{\mu e}\right|<0.3$, at the best. This bound is almost 2 orders of magnitude less stringent than that derived above from the experimental limit on the $\mu \rightarrow e \gamma$ decay. The important difference between these bounds can be understood by noting that while the $\mu \rightarrow$ eee decay is governed by the LFV $H \mu e$ vertex and the SM one Hee, the $\mu \rightarrow e \gamma$ transition is determined by the LFV $H \mu$ e vertex and the SM one $H \mu \mu$. Besides a phase space factor, the branching ratio for the $\mu \rightarrow$ eee decay is suppressed by an additional $\left(m_{e} / m_{\mu}\right)^{2} \approx 2.5 \times 10^{-5}$ factor with respect to the one associated to the two-body $\mu \rightarrow e \gamma$ decay. 


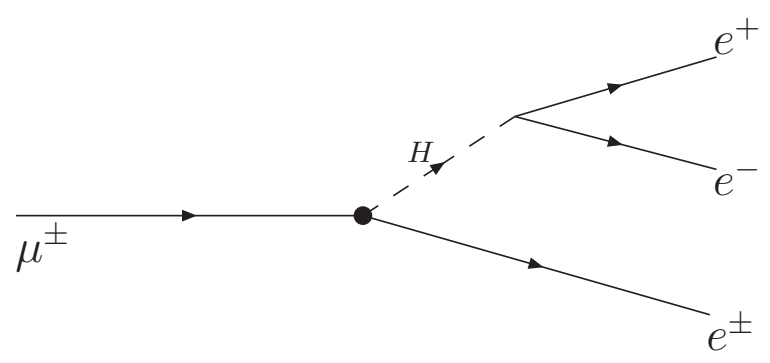

FIG. 4: Diagram contributing to the $\mu \rightarrow e \bar{e} e$ decay.

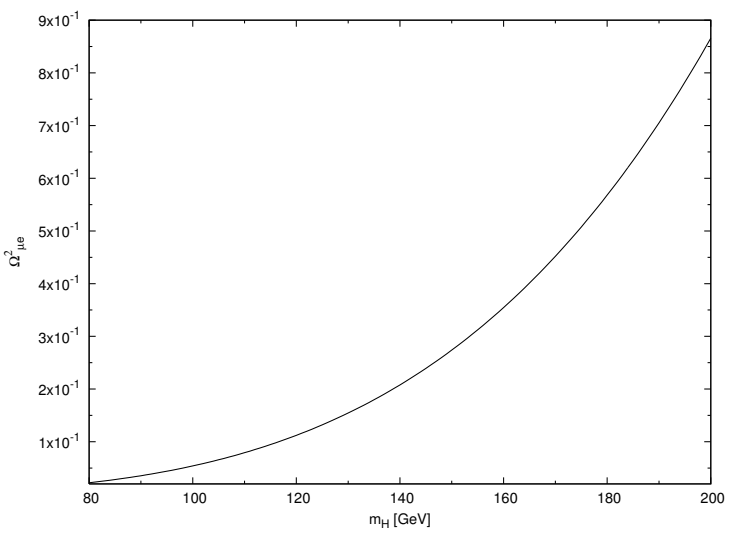

FIG. 5: Behavior of $\left|\Omega_{\mu e}\right|^{2}$ as a function of the Higgs mass.

\section{B. The $l_{i} \rightarrow l_{j} \gamma \gamma$ decay}

The kinematical of the $l_{i} \rightarrow l_{j} \gamma \gamma$ decay is characterized by three dimensionless variables $x, y$, and $z(x+y+z=2)$, which are related to the involved four-vector scalar products as follows:

$$
\begin{aligned}
k_{1} \cdot p_{i} & =-\frac{x m_{i}^{2}}{2}, \\
k_{2} \cdot p_{i} & =-\frac{y m_{i}^{2}}{2}, \\
p_{i} \cdot p_{j} & =-\frac{z m_{i}^{2}}{2}, \\
k_{1} \cdot k_{2} & =\frac{(x+y+\xi-1) m_{i}^{2}}{2}, \\
k_{1} \cdot p_{j} & =\frac{(1-\xi-y) m_{i}^{2}}{2}, \\
k_{2} \cdot p_{j} & =\frac{(1-\xi-x) m_{i}^{2}}{2},
\end{aligned}
$$

where $\xi=m_{j}^{2} / m_{i}^{2}$.

The decay width for the three-body $l_{i} \rightarrow l_{j} \gamma \gamma$ transition is determined by the squared of the amplitude given in Eq. (11). The corresponding branching ratio is given by

$$
\operatorname{Br}\left(l_{i} \rightarrow l_{j} \gamma \gamma\right)=\frac{1}{256 \pi^{3}} \frac{m_{i}}{\Gamma_{i}} \int_{2 \sqrt{\xi}}^{1+\xi} d x \int_{y_{-}}^{y_{+}} d y|\mathcal{M}|^{2},
$$

where $\mathcal{M}=\mathcal{M}^{\mu \nu} \epsilon_{\mu}^{*}\left(k_{1}, \lambda_{1}\right) \epsilon_{\nu}^{*}\left(k_{2}, \lambda_{2}\right)$, with $\epsilon_{\mu}^{*}\left(k_{1}, \lambda_{1}\right)$ and $\epsilon_{\nu}^{*}\left(k_{2}, \lambda_{2}\right)$ the polarization vectors of the photons. The 


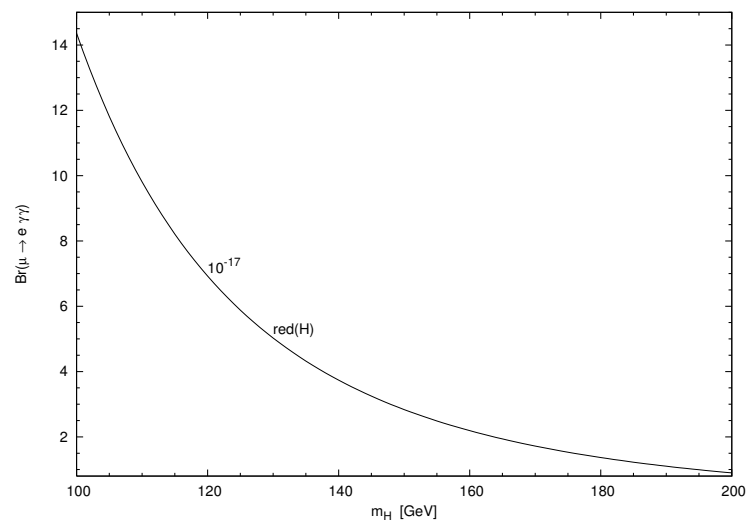

FIG. 6: Behavior of $\operatorname{Br}(\mu \rightarrow e \gamma \gamma)$ as a function of the Higgs mass.

integration limits are given by

$$
\begin{aligned}
& y_{+}=\frac{1}{2}\left[(2-x)+\sqrt{x^{2}-4 \xi}\right], \\
& y_{-}=\frac{1}{2}\left[(2-x)-\sqrt{x^{2}-4 \xi}\right] .
\end{aligned}
$$

The $\tau \rightarrow \mu \gamma \gamma$ decay has already been studied in Ref. [6]. Here, we are interested in obtaining a bound on the $\mu \rightarrow e \gamma \gamma$ transition using the limit on the $\Omega_{\mu e}$ parameter obtained above. The behavior of the corresponding branching ratio is shown in Fig. 6 as a function of the Higgs mass. It can be appreciated from this figure that this branching ratio ranges from approximately $10^{-16}$ to $10^{-17}$. Conservatively, we can assume the following bound:

$$
\operatorname{Br}(\mu \rightarrow e \gamma \gamma)<10^{-16},
$$

which would be the highest branching ratio allowed by the lower bound imposed by LEP [16] on the Higgs mass. This means that the $\mu \rightarrow e \gamma \gamma$ transition may be undetectable if it is mediated by a Higgs boson. This bound for $\operatorname{Br}(\mu \rightarrow e \gamma \gamma)$ is almost 6 orders of magnitude more stringent than the experimental limit reported by the Particle Data Group [15], which is $7.2 \times 10^{-11}$. In obtaining the above constraint, only the contribution of diagrams given in Fig. 2 were considered, as the contribution of the set of diagrams shown in Fig. 1 is insignificant in this process involving very light fermions.

\section{THE CROSS SECTION FOR THE $\gamma \gamma \rightarrow \tau \mu$ PROCESS}

In this section, we analyze the possibility of detecting signals of LFV at the ILC through the $\gamma \gamma \rightarrow \tau \mu$ reaction. The constraint on the $H \mu$ e coupling derived in the previous section from the experimental limit on the $\mu \rightarrow e \gamma$ decay is so restrictive that no signals can be detected via the $\gamma \gamma \rightarrow \mu e$ process. On the other hand, to predict the cross section for the $\gamma \gamma \rightarrow \tau \mu$ reaction, we will need to assume some value for the $\Omega_{\tau \mu}$ parameter. As in the case of the muon decays studied above, we will assume that the imaginary part of $\Omega_{\tau \mu}$ is very suppressed compared with its real part, so from now on $\Omega_{\tau \mu}$ stands for the real part of this parameter. There are various experimental limits [15] which can be used to bound $\Omega_{\tau \mu}$, but the best constraint arises from the anomalous magnetic moment of the muon, which leads to the following bound [14]:

$$
\Omega_{\tau \mu}^{2}<1.1 \times 10^{-3}
$$

for $115 \mathrm{GeV}<m_{H}<200 \mathrm{GeV}$.

The kinematic of the $\gamma \gamma \rightarrow \tau \mu$ process is determined by Mandelstam's variables, which are given by 


$$
\begin{aligned}
& k_{1} \cdot k_{2}=\hat{s} \\
& k_{1} \cdot p_{\mu}=\frac{\hat{t}-m_{\mu}^{2}}{2}, \\
& k_{2} \cdot p_{\mu}=\frac{\hat{u}-m_{\mu}^{2}}{2}, \\
& k_{1} \cdot p_{\tau}=\frac{\hat{u}-m_{\tau}^{2}}{2}, \\
& k_{2} \cdot p_{\tau}=\frac{\hat{t}-m_{\tau}^{2}}{2} .
\end{aligned}
$$

The unpolarized cross section for this process can be written as

$$
\hat{\sigma}(\gamma \gamma \rightarrow \tau \mu)=\frac{1}{16 \pi^{2} \hat{s}^{2}} \int_{\hat{t}_{\min }}^{\hat{t}_{\max }} d \hat{t}|\mathcal{M}|^{2}
$$

where $\mathcal{M}$ is the invariant amplitude which is given by Eq. (11). In the above expression, the integration limits are given by

$$
\hat{t}_{\text {max }}\left(\hat{t}_{\text {min }}\right)=-\frac{\hat{s}}{2}\left(1-\frac{m_{\mu}^{2}+m_{\tau}^{2}}{\hat{s}}\right)(1 \mp \sqrt{1-\delta}),
$$

where

$$
\delta=\left(\frac{2 m_{\mu} m_{\tau}}{\hat{s}-m_{\mu}^{2}-m_{\tau}^{2}}\right)^{2}
$$

On the other hand, the convoluted cross section, i.e., the cross section for the complete process $e^{+} e^{-} \rightarrow \gamma \gamma \rightarrow \tau \mu$, can be written as

$$
\sigma(s)=\int_{\left(m_{\mu}+m_{\tau}\right) / \sqrt{s}}^{y_{\max }} d z \frac{d \mathcal{L}_{\gamma \gamma}}{d z} \hat{\sigma}(\gamma \gamma \rightarrow \tau \mu)
$$

where $\hat{s}=z^{2} s$, with $\sqrt{s}(\sqrt{\hat{s}})$ the center of mass energy of the $e^{+} e^{-}(\gamma \gamma)$ collision, and $\frac{d \mathcal{L}_{\gamma \gamma}}{d z}$ is the luminosity of the photons, defined as

$$
\frac{d \mathcal{L}_{\gamma \gamma}}{d z}=2 z \int_{z^{2} / y_{\max }}^{y_{\max }} \frac{d y}{y} f_{\gamma / e}(y) f_{\gamma / e}\left(z^{2} / y\right)
$$

where the energy spectrum for the backscattered photon is given by [17]

$$
f_{\gamma / e}(y)=\frac{1}{D(\chi)}\left[1-y+\frac{1}{1-y}-\frac{4 y}{\chi(1-y)}+\frac{4 y^{2}}{\chi^{2}(1-y)^{2}}\right]
$$

with

$$
D(\chi)=\left(1-\frac{4}{\chi}-\frac{8}{\chi^{2}}\right) \log (1+\chi)+\frac{1}{2}+\frac{8}{\chi}-\frac{1}{2(1+\chi)^{2}} .
$$

In this expression, $\chi=\left(4 E_{0} \omega_{0}\right) / m_{e}^{2}$, where $m_{e}$ and $E_{0}$ are the mass and energy of the electron, respectively; $\omega_{0}$ is the laser-photon energy; and $y$ represents the fraction of the energy of the incident electron carried by the backscattered photon. The optimum values for the $y_{\max }$ and $\chi$ parameters are $y_{\max } \approx 0.83$ and $\chi=2(1+\sqrt{2})$.

\section{A. Discussion}

We now turn to discuss our results within the experimental context of the ILC. Although this collider is intended to operate initially at a center of mass energy of $500 \mathrm{GeV}$ with a luminosity of $\mathcal{L}_{e^{+} e^{-}}=500 \mathrm{fb}^{-1}$ [1] , it is contemplated 

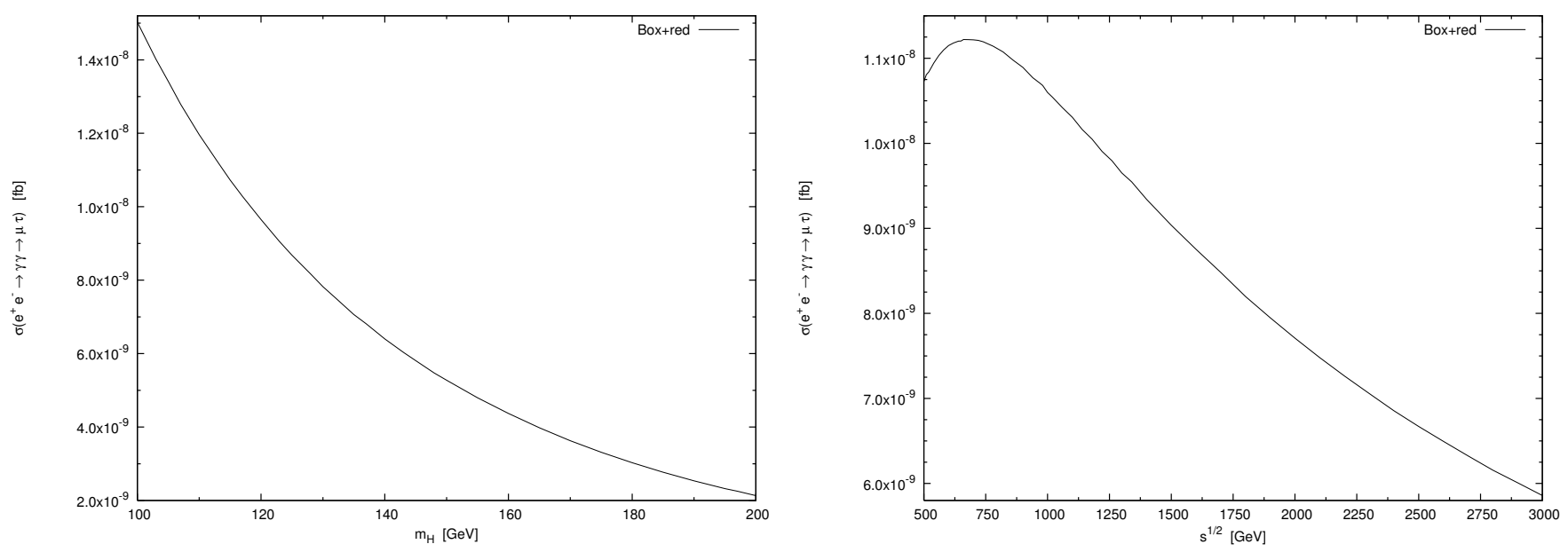

FIG. 7: Contribution of diagrams of Fig. 1 to the cross section $\sigma\left(e^{+} e^{-} \rightarrow \gamma \gamma \rightarrow \mu \tau\right)$ as a function of the Higgs mass for $\sqrt{s}=500 \mathrm{GeV}$ (left) and as a function of $\sqrt{s}$ for $m_{H}=115 \mathrm{GeV}$ (right).

to increase the energy up to about $1 \mathrm{TeV}$ in a subsequent stage. In the following, we will to carry out our numerical analysis in the interval $500 \mathrm{GeV}<\sqrt{s}<3000 \mathrm{GeV}$. We will discuss separately the contributions to this process coming from diagrams displayed in Fig. 1 and Fig. 2, To analyze numerically our results, we will work at first order in the anomalous parameter $\Omega_{\tau \mu}$. We will use the value determined by the upper limit imposed by the anomalous magnetic moment of the muon, namely, $\Omega_{\tau \mu}^{2}<1.1 \times 10^{-3}[14]$. It turns out to be that those contributions coming from diagrams shown in Fig. 1 are proportional to a sum of two amplitudes, each characterized by the factors $\left(m_{\tau} / v\right)\left(\Omega_{\tau \mu}\right)$ and $\left(m_{\mu} / v\right)\left(\Omega_{\tau \mu}\right)$, which determine the intensity of the SM coupling $H l_{i} l_{i}$ and the LFV one $H l_{i} l_{j}$. Such amplitudes represent the two possible situations that can occur when the $\tau$ or $\mu$ particles circulate in the loops. Both such possibilities are implicit in our general and exact calculation represented by the amplitude given by Eq. (11), but we will neglect the contribution associated with a virtual muon, as it is quite suppressed with respect to the corresponding tau contribution.

In Fig. 7 the contribution from diagrams displayed in Fig. 1 is shown as a function of the Higgs mass and the center of mass energy of the collider, whereas in Fig. 8 the contribution arising from diagrams of Fig. 2 are shown. From these figures, it can be appreciated that the contribution from diagrams of Fig. 1 are suppressed by about 7 orders of magnitude compared with the contribution given by diagrams of Fig. 2. For $\sqrt{s}=500 \mathrm{GeV}$, the respective contribution to the cross section can reach a value of $10^{-1} \mathrm{fb}$ for $m_{H} \approx 140 \mathrm{GeV}$, which shows clearly the marginal role played by diagrams of Fig. 1. However, this situation may be different in those processes involving heavier fermions, as the $\gamma \gamma \rightarrow t c$ reaction [10], with $t$ and $c$ the top and charm quarks, respectively. Finally, the number of events as a function of the Higgs mass and the center of mass energy of the collider is presented in Fig. 9. From this figure, it can be appreciated that up to 50 events can be produced with a luminosity of $\mathcal{L}_{e^{+} e^{-}}=500 \mathrm{fb} b^{-1}$.

\section{CONCLUSIONS}

In the minimal standard model the couplings of the Higgs boson to the remaining massive particles are thoroughly determined, which should be considered an outstanding feature of the model. However, there are well-motivated extensions of the model that require the presence of extended Higgs sectors. The extended theory is of course less predictive due to the proliferation of free parameters. The bonus is the appearance of interesting new physics effects such as lepton flavor violating transitions that are mediated by the physical scalars of the theory. In this paper, the potential of extended Yukawa sectors in generating flavor violation both in the lepton and the quark sectors was explored in a model independent manner using the effective Lagrangian approach. A Yukawa sector that includes $S U_{L}(2) \times U_{Y}(1)$-invariant operators of up to dimension-six, which reproduces the main features of extended Yukawa sectors, was considered. The one-loop contribution of a flavor violating $H f_{i} f_{j}$ coupling, where $f_{i}$ and $f_{j}$ stand for charged leptons or quarks, to the on-shell $\gamma f_{i} f_{j}$ and $\gamma \gamma f_{i} f_{j}$ electromagnetic interactions was calculated. Exact formulae for the amplitudes associated with these couplings were presented and used to investigate electromagnetic lepton flavor violating transitions. The experimental limit on the branching ratio for the $\mu \rightarrow e \gamma$ decay was used to impose the bound $\left|\Omega_{\mu e}\right|<7 \times 10^{-3}$ on the $H \mu e$ coupling. This bound allowed us to derive the limit $\operatorname{Br}(\mu \rightarrow e \gamma \gamma)<10^{-16}$, which is about 6 orders of magnitude more stringent than the corresponding experimental 

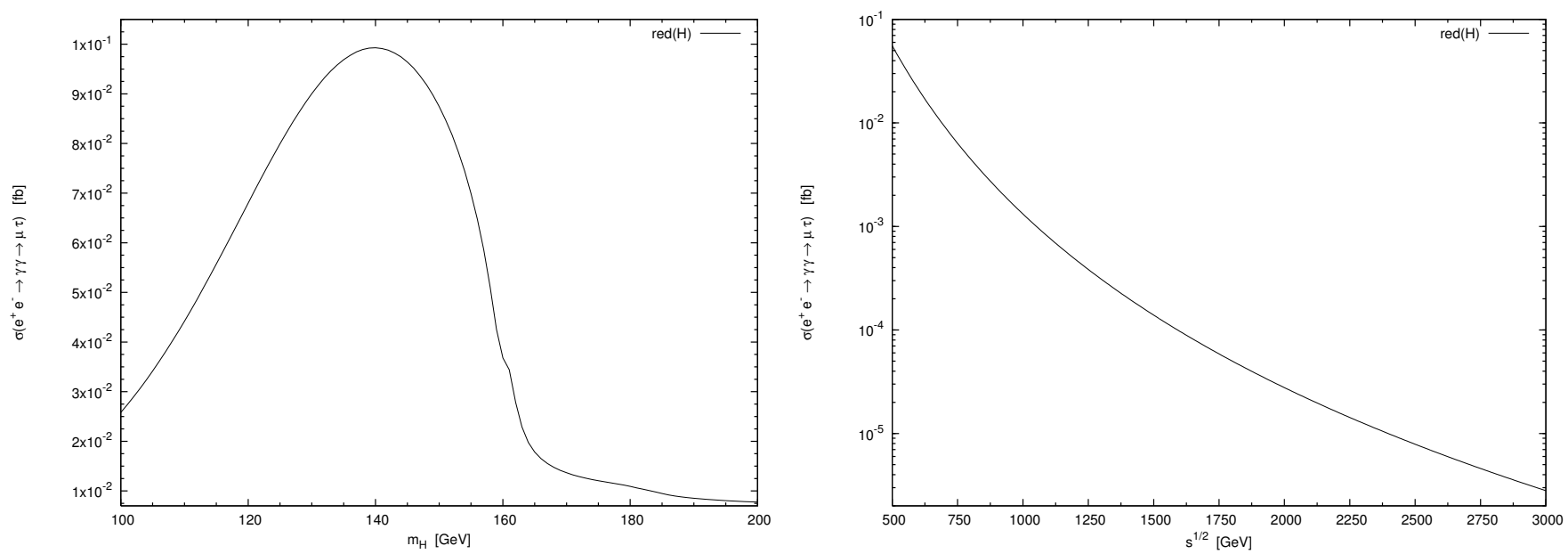

FIG. 8: Contribution of diagrams of Fig. 2 to the cross section $\sigma\left(e^{+} e^{-} \rightarrow \gamma \gamma \rightarrow \mu \tau\right)$ as a function of the Higgs mass for $\sqrt{s}=500 \mathrm{GeV}$ (left) and as a function of $\sqrt{s}$ for $m_{H}=115 \mathrm{GeV}$ (right).
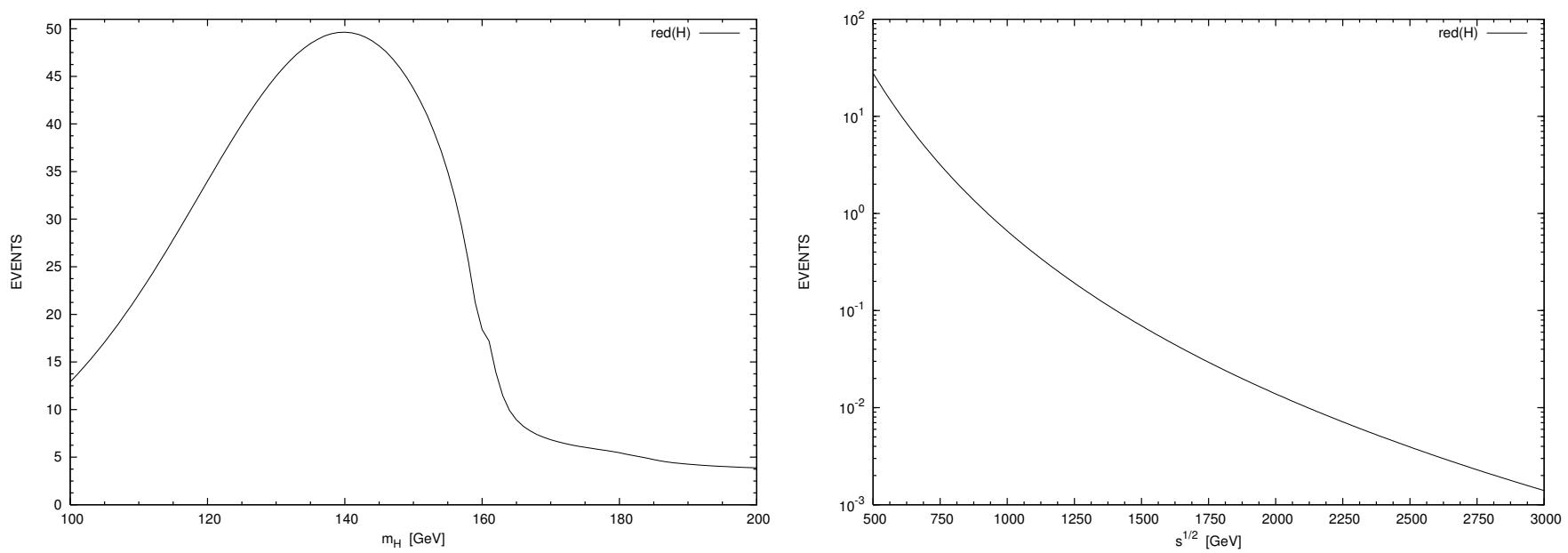

FIG. 9: The number of events as a function of the Higgs mass for $\sqrt{s}=500 \mathrm{GeV}$ (left) and as a function of $\sqrt{s}$ for $m_{H}=115$ $\mathrm{GeV}$ (right)..

limit. The possibility of detecting signals of lepton flavor violation at the ILC through the reaction $e^{+} e^{-} \rightarrow \gamma \gamma \rightarrow \tau \mu$ was explored. It was found that with the projected luminosity of this machine up to half a hundred of $\tau^{ \pm} \mu^{\mp}$ events could be produced. Although in some processes this number of events could be insufficient to detect the signal, it is important to have in mind that the $\tau^{ \pm} \mu^{\mp}$ signal is a clean environment and is spectacular in the sense that the process is free of background, having thus the chance of being observed.

\section{Acknowledgments}

We acknowledge financial support from CONACYT and SNI (México).

\section{APPENDIX: FORM FACTORS OF THE $\gamma \gamma f_{i} f_{j}$ COUPLING}

In this appendix, we present the expressions for the form factors $F_{n_{R, L}}$. We present results only for the righthanded form factors $F_{n_{R}}$, as it can be passed to the left-handed ones via the interchanges $\omega_{k l} \omega_{m k} \leftrightarrow \omega_{k l}^{*} \omega_{m k}^{*}$ and $\omega_{k l}^{*} \omega_{m k} \leftrightarrow \omega_{k l} \omega_{m k}^{*}$. There are a total of 20 form factors of this class, but they are linked by the Bose symmetry, so one needs to list only 11 of them, the rest being obtained through the interchange $k_{1} \leftrightarrow k_{2}$. Also, the arguments of 


\begin{tabular}{ccccccccccc}
\hline$(j)$ & 1 & 2 & 3 & 4 & 5 & 6 & 7 & 8 & 9 & 10 \\
\hline$B_{0}\left(i_{0}\right)$ & 0 & $m_{H}^{2}$ & $m_{H}^{2}$ & & & & & & \\
$B_{0}\left(i_{1}\right)$ & 0 & $m_{H}^{2}$ & $m_{k}^{2}$ & & & & & & \\
$B_{0}\left(i_{2}\right)$ & 0 & $m_{k}^{2}$ & $m_{k}^{2}$ & & & & & & \\
$B_{0}\left(i_{3}\right)$ & $m_{j}^{2}$ & $m_{H}^{2}$ & $m_{k}^{2}$ & & & & & & \\
$B_{0}\left(i_{4}\right)$ & $m_{j}^{2}+h\left(k_{1}, k_{2}\right)$ & $m_{H}^{2}$ & $m_{k}^{2}$ & & & & & & \\
$B_{0}(1)$ & $m_{j}^{2}+2 k_{1} \cdot p_{j}$ & $m_{H}^{2}$ & $m_{k}^{2}$ & & & & & & \\
$B_{0}(2)$ & $m_{j}^{2}+2 k_{2} \cdot p_{j}$ & $m_{H}^{2}$ & $m_{k}^{2}$ & & & & & & \\
$C_{0}(i)$ & 0 & 0 & $2 k_{1} \cdot k_{2}$ & $m_{W}^{2}$ & $m_{W}^{2}$ & $m_{W}^{2}$ & & & \\
$C_{0}(I I)$ & 0 & 0 & $2 k_{1} \cdot k_{2}$ & $m_{t}^{2}$ & $m_{t}^{2}$ & $m_{t}^{2}$ & & & \\
$C_{0}(1)$ & 0 & $m_{j}^{2}$ & $m_{j}^{2}+2 k_{1} \cdot p_{j}$ & $m_{k}^{2}$ & $m_{k}^{2}$ & $m_{H}^{2}$ & & & \\
$C_{0}(2)$ & 0 & $m_{j}^{2}$ & $m_{j}^{2}+2 k_{2} \cdot p_{j}$ & $m_{k}^{2}$ & $m_{k}^{2}$ & $m_{H}^{2}$ & & & & \\
$C_{0}(3)$ & 0 & $m_{j}^{2}+2 k_{1} \cdot p_{j}$ & $m_{j}^{2}+h\left(k_{1}, k_{2}\right)$ & $m_{k}^{2}$ & $m_{k}^{2}$ & $m_{H}^{2}$ & & & \\
$C_{0}(4)$ & 0 & $m_{j}^{2}+2 k_{2} \cdot p_{j}$ & $m_{j}^{2}+h\left(k_{1}, k_{2}\right)$ & $m_{k}^{2}$ & $m_{k}^{2}$ & $m_{H}^{2}$ & & & & \\
$D_{0}(1)$ & $2 k_{1} \cdot k_{2}$ & $m_{j}^{2}+h\left(k_{1}, k_{2}\right)$ & $m_{j}^{2}+2 k_{1} \cdot p_{j}$ & 0 & $m_{j}^{2}$ & 0 & $m_{k}^{2}$ & $m_{k}^{2}$ & $m_{H}^{2}$ & $m_{k}^{2}$ \\
$D_{0}(2)$ & $2 k_{1} \cdot k_{2}$ & $m_{j}^{2}+h\left(k_{1}, k_{2}\right)$ & $m_{j}^{2}+2 k_{2} \cdot p_{j}$ & 0 & $m_{j}^{2}$ & 0 & $m_{k}^{2}$ & $m_{k}^{2}$ & $m_{H}^{2}$ & $m_{k}^{2}$ \\
\hline
\end{tabular}

TABLE I: Arguments for the Passarino-Veltman scalar functions. The expression has been defined as: $h\left(k_{1}, k_{2}\right)=2\left(k_{1} \cdot k_{2}+\right.$ $\left.\left(k_{1}+k_{2}\right) \cdot p_{j}\right)$.

the diverse Passarino-Veltman coefficients are listed in Tables I, II, III y IV These form factors are given by:

$$
\begin{aligned}
F_{1_{R}}= & 4 \omega_{i k} m_{W}\left(m_{k} \omega_{k j}\left(D_{00}(1)+D_{00}(2)\right)-m_{j} \omega_{k j}^{*}\left(D_{002}(1)+D_{002}(2)\right)\right) \\
F_{2_{R}}= & \frac{\omega_{i k} m_{W}^{3}}{2}\left\{8\left(m_{k} \omega_{k j}\left(D_{22}(1)+D_{22}(2)\right)-m_{j} \omega_{k j}^{*}\left(D_{222}(1)+D_{222}(2)\right)\right)+\frac{1}{g\left(k_{1}, k_{2}\right)}\left[m_{j}\left(m_{H}^{2}-m_{k}^{2}\right) \omega_{k j}^{*}\right.\right. \\
& \times\left(k_{1}+k_{2}\right) \cdot p_{j} B_{0}\left(i_{1}\right)+4\left(k_{1} \cdot k_{2}+\left(k_{1}+k_{2}\right) \cdot p_{j}\right)\left(m_{j}^{2}+2\left(k_{1} \cdot k_{2}+\left(k_{1}+k_{2}\right) \cdot p_{j}\right)\right)\left(\left(m _ { k } \omega _ { k j } \left(C_{0}(4)\right.\right.\right. \\
& \left.\left.+C_{1}(6)+C_{2}(6)\right)+m_{j} \omega_{k j}^{*}\left(C_{0}(4)+2 C_{1}(6)+C_{11}(4)+2\left(C_{12}(4)+C_{2}(6)\right)+C_{22}(4)\right)\right) k_{1} \cdot p_{j} \\
& +\left(m_{k} \omega_{k j}\left(C_{0}(3)+C_{1}(5)+C_{2}(5)\right)+m_{j} \omega_{k j}^{*}\left(C_{0}(3)+2 C_{1}(5)+C_{11}(3)+2\left(C_{12}(3)+C_{2}(5)\right)+C_{22}(3)\right)\right) \\
& \left.\times k_{2} \cdot p_{j}\right)+B_{0}\left(i_{4}\right)\left(k_{1}+k_{2}\right) \cdot p_{j}\left(m_{j}\left(\left(m_{j}^{2}+m_{k}^{2}-m_{H}^{2}\right) \omega_{k j}^{*}+2 m_{j} m_{k} \omega_{k j}\right)+2\left(m_{j} \omega_{k j}^{*}+2 m_{k} \omega_{k j}\right)\right. \\
& \left.\left.\left.\times\left(k_{1} \cdot k_{2}+\left(k_{1}+k_{2}\right) \cdot p_{j}\right)\right)\right]\right\} \\
F_{3_{R}}= & -4 \omega_{k j}^{*} \omega_{i k} m_{W}^{2}\left(D_{00}(1)+D_{001}(1)+D_{001}(2)+D_{003}(1)\right),
\end{aligned}
$$

where

$$
g\left(k_{1}, k_{2}\right)=k_{1} \cdot p_{j} k_{2} \cdot p_{j}\left(k_{1} \cdot k_{2}+\left(k_{1}+k_{2}\right) \cdot p_{j}\right)\left(m_{j}^{2}+2\left(k_{1} \cdot k_{2}+\left(k_{1}+k_{2}\right) \cdot p_{j}\right)\right),
$$




\begin{tabular}{|c|c|c|c|c|c|c|c|c|c|c|}
\hline$(j)$ & 1 & 2 & 3 & 4 & 5 & 6 & 7 & 8 & 9 & 10 \\
\hline$\overline{C_{1}\left(i_{0}\right)}$ & $m_{j}^{2}+h\left(k_{1}, k_{2}\right)$ & $2 k_{1} \cdot k_{2}$ & $m_{j}^{2}$ & $m_{H}^{2}$ & $m_{k}^{2}$ & $m_{k}^{2}$ & & & & \\
\hline$C_{1}(1)$ & $m_{j}^{2}$ & 0 & $m_{j}^{2}+2 k_{1} \cdot p_{j}$ & $m_{H}^{2}$ & $m_{k}^{2}$ & $m_{k}^{2}$ & & & & \\
\hline$C_{1}(2)$ & $m_{j}^{2}$ & 0 & $m_{j}^{2}+2 k_{2} \cdot p_{j}$ & $m_{H}^{2}$ & $m_{k}^{2}$ & $m_{k}^{2}$ & & & & \\
\hline$C_{1}(3)$ & $m_{j}^{2}+2 k_{1} \cdot p_{j}$ & 0 & $m_{j}^{2}$ & $m_{H}^{2}$ & $m_{k}^{2}$ & $m_{k}^{2}$ & & & & \\
\hline$C_{1}(4)$ & $m_{j}^{2}+2 k_{2} \cdot p_{j}$ & 0 & $m_{j}^{2}$ & $m_{H}^{2}$ & $m_{k}^{2}$ & $m_{k}^{2}$ & & & & \\
\hline$C_{1}(5)$ & $m_{j}^{2}+2 k_{1} \cdot p_{j}$ & 0 & $m_{j}^{2}+h\left(k_{1}, k_{2}\right)$ & $m_{H}^{2}$ & $m_{k}^{2}$ & $m_{k}^{2}$ & & & & \\
\hline$C_{1}(6)$ & $m_{j}^{2}+2 k_{2} \cdot p_{j}$ & 0 & $m_{j}^{2}+h\left(k_{1}, k_{2}\right)$ & $m_{H}^{2}$ & $m_{k}^{2}$ & $m_{k}^{2}$ & & & & \\
\hline$C_{2}(1)$ & $m_{j}^{2}$ & 0 & $m_{j}^{2}+2 k_{1} \cdot p_{j}$ & $m_{H}^{2}$ & $m_{k}^{2}$ & $m_{k}^{2}$ & & & & \\
\hline$C_{2}(2)$ & $m_{j}^{2}$ & 0 & $m_{j}^{2}+2 k_{2} \cdot p_{j}$ & $m_{H}^{2}$ & $m_{k}^{2}$ & $m_{k}^{2}$ & & & & \\
\hline$C_{2}(3)$ & $m_{j}^{2}+2 k_{1} \cdot p_{j}$ & 0 & $m_{j}^{2}$ & $m_{H}^{2}$ & $m_{k}^{2}$ & $m_{k}^{2}$ & & & & \\
\hline$C_{2}(4)$ & $m_{j}^{2}+2 k_{2} \cdot p_{j}$ & 0 & $m_{j}^{2}$ & $m_{H}^{2}$ & $m_{k}^{2}$ & $m_{k}^{2}$ & & & & \\
\hline$C_{2}(5)$ & $m_{j}^{2}+2 k_{1} \cdot p_{j}$ & 0 & $m_{j}^{2}+h\left(k_{1}, k_{2}\right)$ & $m_{H}^{2}$ & $m_{k}^{2}$ & $m_{k}^{2}$ & & & & \\
\hline$C_{2}(6)$ & $m_{j}^{2}+2 k_{2} \cdot p_{j}$ & 0 & $m_{j}^{2}+h\left(k_{1}, k_{2}\right)$ & $m_{H}^{2}$ & $m_{k}^{2}$ & $m_{k}^{2}$ & & & & \\
\hline$D_{1}(1)$ & $2 k_{1} \cdot k_{2}$ & $m_{j}^{2}+h\left(k_{1}, k_{2}\right)$ & $m_{j}^{2}+2 k_{1} \cdot p_{j}$ & 0 & $m_{j}^{2}$ & 0 & $m_{k}^{2}$ & $m_{k}^{2}$ & $m_{H}^{2}$ & $m_{k}^{2}$ \\
\hline$D_{1}(2)$ & $2 k_{1} \cdot k_{2}$ & $m_{j}^{2}+h\left(k_{1}, k_{2}\right)$ & $m_{j}^{2}+2 k_{2} \cdot p_{j}$ & 0 & $m_{j}^{2}$ & 0 & $m_{k}^{2}$ & $m_{k}^{2}$ & $m_{H}^{2}$ & $m_{k}^{2}$ \\
\hline$D_{2}(1)$ & $2 k_{1} \cdot k_{2}$ & $m_{j}^{2}+h\left(k_{1}, k_{2}\right)$ & $m_{j}^{2}+2 k_{1} \cdot p_{j}$ & 0 & $m_{j}^{2}$ & 0 & $m_{k}^{2}$ & $m_{k}^{2}$ & $m_{H}^{2}$ & $m_{k}^{2}$ \\
\hline$D_{2}(2)$ & $2 k_{1} \cdot k_{2}$ & $m_{j}^{2}+h\left(k_{1}, k_{2}\right)$ & $m_{j}^{2}+2 k_{2} \cdot p_{j}$ & 0 & $m_{j}^{2}$ & 0 & $m_{k}^{2}$ & $m_{k}^{2}$ & $m_{H}^{2}$ & $m_{k}^{2}$ \\
\hline
\end{tabular}

TABLE II: Arguments for the Passarino-Veltman tensorial coefficients with an index.

\begin{tabular}{|c|c|c|c|c|c|c|c|c|c|c|}
\hline$(j)$ & 1 & 2 & 3 & 4 & 5 & 6 & 7 & 8 & 9 & 10 \\
\hline$\overline{C_{00}(1)}$ & $m_{j}^{2}+2 k_{1} \cdot p_{j}$ & 0 & $\overline{m_{j}^{2}}$ & $\overline{m_{H}^{2}}$ & $m_{k}^{2}$ & $\overline{m_{k}^{2}}$ & & & & \\
\hline$C_{00}(2)$ & $m_{j}^{2}+2 k_{2} \cdot p_{j}$ & 0 & $m_{j}^{2}$ & $m_{H}^{2}$ & $m_{k}^{2}$ & $m_{k}^{2}$ & & & & \\
\hline$C_{00}(3)$ & $m_{j}^{2}+2 k_{1} \cdot p_{j}$ & 0 & $m_{j}^{2}+h\left(k_{1}, k_{2}\right)$ & $m_{H}^{2}$ & $m_{k}^{2}$ & $m_{k}^{2}$ & & & & \\
\hline$C_{00}(4)$ & $m_{j}^{2}+2 k_{2} \cdot p_{j}$ & 0 & $m_{j}^{2}+h\left(k_{1}, k_{2}\right)$ & $m_{H}^{2}$ & $m_{k}^{2}$ & $m_{k}^{2}$ & & & & \\
\hline$C_{11}(1)$ & $m_{j}^{2}+2 k_{1} \cdot p_{j}$ & 0 & $m_{j}^{2}$ & $m_{H}^{2}$ & $m_{k}^{2}$ & $m_{k}^{2}$ & & & & \\
\hline$C_{11}(2)$ & $m_{j}^{2}+2 k_{2} \cdot p_{j}$ & 0 & $m_{j}^{2}$ & $m_{H}^{2}$ & $m_{k}^{2}$ & $m_{k}^{2}$ & & & & \\
\hline$C_{11}(3)$ & $m_{j}^{2}+2 k_{1} \cdot p_{j}$ & 0 & $m_{j}^{2}+h\left(k_{1}, k_{2}\right)$ & $m_{H}^{2}$ & $m_{k}^{2}$ & $m_{k}^{2}$ & & & & \\
\hline$C_{11}(4)$ & $m_{j}^{2}+2 k_{2} \cdot p_{j}$ & 0 & $m_{j}^{2}+h\left(k_{1}, k_{2}\right)$ & $m_{H}^{2}$ & $m_{k}^{2}$ & $m_{k}^{2}$ & & & & \\
\hline$C_{12}(1)$ & $m_{j}^{2}+2 k_{1} \cdot p_{j}$ & 0 & $m_{j}^{2}$ & $m_{H}^{2}$ & $m_{k}^{2}$ & $m_{k}^{2}$ & & & & \\
\hline$C_{12}(2)$ & $m_{j}^{2}+2 k_{2} \cdot p_{j}$ & 0 & $m_{j}^{2}$ & $m_{H}^{2}$ & $m_{k}^{2}$ & $m_{k}^{2}$ & & & & \\
\hline$C_{12}(3)$ & $m_{j}^{2}+2 k_{1} \cdot p_{j}$ & 0 & $m_{j}^{2}+h\left(k_{1}, k_{2}\right)$ & $m_{H}^{2}$ & $m_{k}^{2}$ & $m_{k}^{2}$ & & & & \\
\hline$C_{12}(4)$ & $m_{j}^{2}+2 k_{2} \cdot p_{j}$ & 0 & $m_{j}^{2}+h\left(k_{1}, k_{2}\right)$ & $m_{H}^{2}$ & $m_{k}^{2}$ & $m_{k}^{2}$ & & & & \\
\hline$C_{22}(1)$ & $m_{j}^{2}+2 k_{1} \cdot p_{j}$ & 0 & $m_{j}^{2}$ & $m_{H}^{2}$ & $m_{k}^{2}$ & $m_{k}^{2}$ & & & & \\
\hline$C_{22}(2)$ & $m_{j}^{2}+2 k_{2} \cdot p_{j}$ & 0 & $m_{j}^{2}$ & $m_{H}^{2}$ & $m_{k}^{2}$ & $m_{k}^{2}$ & & & & \\
\hline$C_{22}(3)$ & $m_{j}^{2}+2 k_{1} \cdot p_{j}$ & 0 & $m_{j}^{2}+h\left(k_{1}, k_{2}\right)$ & $m_{H}^{2}$ & $m_{k}^{2}$ & $m_{k}^{2}$ & & & & \\
\hline$C_{22}(4)$ & $m_{j}^{2}+2 k_{2} \cdot p_{j}$ & 0 & $m_{j}^{2}+h\left(k_{1}, k_{2}\right)$ & $m_{H}^{2}$ & $m_{k}^{2}$ & $m_{k}^{2}$ & & & & \\
\hline$D_{00}(1)$ & $2 k_{1} \cdot k_{2}$ & $m_{j}^{2}+h\left(k_{1}, k_{2}\right)$ & $m_{j}^{2}+2 k_{1} \cdot p_{j}$ & 0 & $m_{j}^{2}$ & 0 & $m_{k}^{2}$ & $m_{k}^{2}$ & $m_{H}^{2}$ & $m_{k}^{2}$ \\
\hline$D_{00}(2)$ & $2 k_{1} \cdot k_{2}$ & $m_{j}^{2}+h\left(k_{1}, k_{2}\right)$ & $m_{j}^{2}+2 k_{2} \cdot p_{j}$ & 0 & $m_{j}^{2}$ & 0 & $m_{k}^{2}$ & $m_{k}^{2}$ & $m_{H}^{2}$ & $m_{k}^{2}$ \\
\hline$D_{12}(1)$ & $2 k_{1} \cdot k_{2}$ & $m_{j}^{2}+h\left(k_{1}, k_{2}\right)$ & $m_{j}^{2}+2 k_{1} \cdot p_{j}$ & 0 & $m_{j}^{2}$ & 0 & $m_{k}^{2}$ & $m_{k}^{2}$ & $m_{H}^{2}$ & $m_{k}^{2}$ \\
\hline$D_{12}(2)$ & $2 k_{1} \cdot k_{2}$ & $m_{j}^{2}+h\left(k_{1}, k_{2}\right)$ & $m_{j}^{2}+2 k_{2} \cdot p_{j}$ & 0 & $m_{j}^{2}$ & 0 & $m_{k}^{2}$ & $m_{k}^{2}$ & $m_{H}^{2}$ & $m_{k}^{2}$ \\
\hline$D_{22}(1)$ & $2 k_{1} \cdot k_{2}$ & $m_{j}^{2}+h\left(k_{1}, k_{2}\right)$ & $m_{j}^{2}+2 k_{1} \cdot p_{j}$ & 0 & $m_{j}^{2}$ & 0 & $m_{k}^{2}$ & $m_{k}^{2}$ & $m_{H}^{2}$ & $m_{k}^{2}$ \\
\hline$D_{22}(2)$ & $2 k_{1} \cdot k_{2}$ & $m_{j}^{2}+h\left(k_{1}, k_{2}\right)$ & $m_{j}^{2}+2 k_{2} \cdot p_{j}$ & 0 & $m_{j}^{2}$ & 0 & $m_{k}^{2}$ & $m_{k}^{2}$ & $m_{H}^{2}$ & $m_{k}^{2}$ \\
\hline$D_{23}(1)$ & $2 k_{1} \cdot k_{2}$ & $m_{j}^{2}+h\left(k_{1}, k_{2}\right)$ & $m_{j}^{2}+2 k_{1} \cdot p_{j}$ & 0 & $m_{j}^{2}$ & 0 & $m_{k}^{2}$ & $m_{k}^{2}$ & $m_{H}^{2}$ & $m_{k}^{2}$ \\
\hline$D_{23}(2)$ & $2 k_{1} \cdot k_{2}$ & $m_{j}^{2}+h\left(k_{1}, k_{2}\right)$ & $m_{j}^{2}+2 k_{2} \cdot p_{j}$ & 0 & $m_{j}^{2}$ & 0 & $m_{k}^{2}$ & $m_{k}^{2}$ & $m_{H}^{2}$ & $m_{k}^{2}$ \\
\hline
\end{tabular}

TABLE III: Arguments for the Passarino-Veltman tensorial coefficients with two indices. 


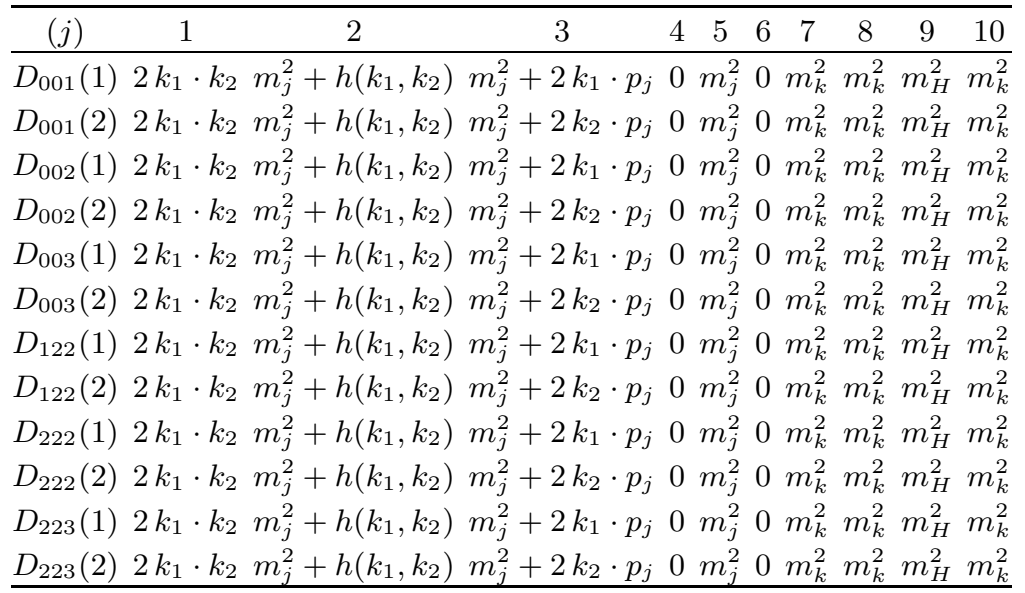

TABLE IV: Arguments for the Passarino-Veltman tensorial coefficients with three indices.

$$
\begin{aligned}
& F_{5_{R}}=m_{W}^{2}\left\{2 \omega _ { k j } ^ { * } \omega _ { i k } \left(C_{0}(3)+2 C_{1}(5)+C_{11}(3)+2 C_{12}(3)+2 C_{2}(5)+C_{22}(3)+2\left(D_{002}(1)+D_{002}(2)\right)\right.\right. \\
& \left.-2 D_{12}(1) k_{1} \cdot k_{2}\right)+\frac{\omega_{k j}^{*} \omega_{i k}}{k_{1} \cdot p_{j}}\left[2 C_{00}(3)+m_{H}^{2}\left(C_{0}(3)+C_{1}(5)+C_{2}(5)\right)-m_{k}^{2}\left(C_{0}(3)+C_{1}(5)+C_{2}(5)\right)\right. \\
& +m_{j}^{2}\left(C_{0}(3)+3 C_{1}(5)+2 C_{11}(3)+4 C_{12}(3)+3 C_{2}(5)+2 C_{22}(3)\right)+2\left(C_{12}(3)+C_{2}(5)+C_{22}(3)\right)\left(k_{1} \cdot k_{2}\right. \\
& \left.\left.+k_{2} \cdot p_{j}\right)\right]+\frac{\omega_{k j}^{*} \omega_{i k}\left(B_{0}\left(i_{4}\right)-2 C_{00}(4)\right)}{k_{2} \cdot p_{j}}-\frac{\omega_{k j}^{*} \omega_{i k}\left(m_{H}^{2} B_{0}\left(i_{0}\right)-\left(m_{H}^{2}-m_{k}^{2}\right)\left(B_{0}\left(i_{1}\right)-1\right)-m_{k}^{2} B_{0}\left(i_{2}\right)\right)}{2 k_{1} \cdot p_{j}\left(k_{1} \cdot k_{2}+\left(k_{1}+k_{2}\right) \cdot p_{j}\right)} \\
& -\frac{\omega_{k j}^{*} \omega_{i k}\left(m_{H}^{2}-m_{k}^{2}+m_{H}^{2} B_{0}\left(i_{0}\right)-m_{k}^{2} B_{0}\left(i_{2}\right)\right)}{\left(m_{j}^{2}-m_{i}^{2}\right) k_{2} \cdot p_{j}}+\frac{1}{m_{j}\left(m_{j}^{2}-m_{i}^{2}\right)\left(m_{j}^{2}-m_{i}^{2}+2 k_{2} \cdot p_{j}\right)}\left[\left(m_{H}^{2}-m_{k}^{2}\right)\right. \\
& \times\left(m_{j} \omega_{k j}^{*} \omega_{i k}-m_{i} \omega_{i k}^{*} \omega_{k j}\right) B_{0}\left(i_{1}\right)-\left(m_{j} \omega_{k j}^{*}\left(2 m_{i} m_{k} \omega_{i k}^{*}+\left(m_{j}^{2}+m_{k}^{2}-m_{H}^{2}\right) \omega_{i k}\right)+\left(m_{i}\left(m_{j}^{2}+m_{k}^{2}\right) \omega_{i k}^{*}\right.\right. \\
& \left.\left.-m_{H}^{2} m_{i} \omega_{i k}^{*}+2 m_{j}^{2} m_{k} \omega_{i k}\right) \omega_{k j}\right) B_{0}\left(i_{3}\right)+2 m_{j}\left(m_{j}^{2}-m_{i}^{2}\right)\left(\omega _ { i k } \left(m_{H}^{2} \omega_{k j}^{*}\left(C_{0}(2)+C_{1}(4)+C_{2}(4)\right)\right.\right. \\
& -m_{k}^{2} \omega_{k j}^{*}\left(C_{0}(2)+C_{1}(4)+C_{2}(4)\right)-m_{j} m_{k} \omega_{k j}\left(C_{0}(2)+C_{1}(4)+C_{2}(4)\right)+m_{j}^{2} \omega_{k j}^{*}\left(C_{1}(2)+C_{11}(2)+2 C_{12}(2)\right. \\
& \left.\left.+C_{2}(2)+C_{22}(2)\right)\right)-m_{i} \omega_{i k}^{*}\left(m_{k} \omega_{k j}^{*}\left(C_{0}(2)+C_{1}(4)+C_{2}(4)\right)+m_{j} \omega_{k j}\left(C_{0}(2)+C_{1}(2)+C_{1}(4)+C_{11}(2)\right.\right. \\
& \left.\left.\left.\left.+2 C_{12}(2)+C_{2}(2)+C_{2}(4)+C_{22}(2)\right)\right)\right)\right]+\frac{1}{2 k_{2} \cdot p_{j}\left(m_{j}^{2}+2 k_{2} \cdot p_{j}\right)\left(m_{j}^{2}-m_{i}^{2}+2 k_{2} \cdot p_{j}\right)}\left[m _ { j } \left(m_{j} \omega_{k j}^{*}\right.\right. \\
& \left.\times\left(\left(m_{H}^{2}-m_{j}^{2}-m_{k}^{2}\right) \omega_{i k}-2 m_{i} m_{k} \omega_{i k}^{*}\right)+\left(m_{H}^{2} m_{i} \omega_{i k}^{*}-m_{i}\left(m_{j}^{2}+m_{k}^{2}\right) \omega_{i k}^{*}-2 m_{j}^{2} m_{k} \omega_{i k}\right) \omega_{k j}\right) B_{0}(2) \\
& +m_{j}\left(m_{H}^{2}-m_{k}^{2}\right)\left(m_{j} \omega_{k j}^{*} \omega_{i k}-m_{i} \omega_{i k}^{*} \omega_{k j}\right) B_{0}\left(i_{1}\right)-2\left(2 m_{i} m_{k} \omega_{i k}^{*} \omega_{k j}^{*}+\left(m_{j}^{2}+2 m_{k}^{2}-2 m_{H}^{2}\right) \omega_{k j}^{*} \omega_{i k}\right. \\
& \left.\left.+m_{j}\left(m_{i} \omega_{i k}^{*}+2 m_{k} \omega_{i k}\right) \omega_{k j}\right) k_{2} \cdot p_{j} B_{0}(2)\right]-\frac{k_{1} \cdot k_{2}+\left(k_{1}+k_{2}\right) \cdot p_{j}}{2 g\left(k_{1}, k_{2}\right)}\left[\omega _ { k j } ^ { * } \omega _ { i k } ( k _ { 1 } + k _ { 2 } ) \cdot p _ { j } \left(\left(m_{H}^{2}-m_{k}^{2}\right)\right.\right. \\
& \left.\left.\left.\times B_{0}\left(i_{1}\right)+B_{0}\left(i_{4}\right)\left(m_{j}^{2}+m_{k}^{2}-m_{H}^{2}+2\left(k_{1} \cdot k_{2}+\left(k_{1}+k_{2}\right) \cdot p_{j}\right)\right)\right)\right]\right\} \\
& F_{7_{R}}=-\frac{2 \omega_{k j}^{*} \omega_{i k} m_{W}^{4}}{k_{2} \cdot p_{j}}\left\{C_{12}(4)+C_{2}(6)+C_{22}(4)+2\left(D_{122}(1)+D_{122}(2)+D_{223}(1)\right) k_{2} \cdot p_{j}\right\} \text {, }
\end{aligned}
$$




$$
\begin{aligned}
& F_{9_{R}}=\frac{\omega_{i k} m_{W}^{3}}{4}\left\{8\left(m_{k} \omega_{k j}\left(D_{2}(1)+D_{2}(2)\right)-m_{j} \omega_{k j}^{*}\left(D_{22}(1)+D_{22}(2)\right)\right)-\frac{1}{g\left(k_{1}, k_{2}\right)}\left[m_{j}\left(m_{H}^{2}-m_{k}^{2}\right) \omega_{k j}^{*}\right.\right. \\
& \times\left(k_{1}+k_{2}\right) \cdot p_{j} B_{0}\left(i_{1}\right)+4\left(k_{1} \cdot k_{2}+\left(k_{1}+k_{2}\right) \cdot p_{j}\right)\left(m_{j}^{2}+2\left(k_{1} \cdot k_{2}+\left(k_{1}+k_{2}\right) \cdot p_{j}\right)\right)\left(\left(m_{k} \omega_{k j} C_{0}(4)\right.\right. \\
& \left.+m_{j} \omega_{k j}^{*}\left(C_{0}(4)+C_{1}(6)+C_{2}(6)\right)\right) k_{1} \cdot p_{j}+\left(m_{k} \omega_{k j}\left(C_{0}(3)+C_{1}(5)+C_{2}(5)\right)+m_{j} \omega_{k j}^{*}\left(C_{0}(3)+2 C_{1}(5)\right.\right. \\
& \left.\left.\left.+C_{11}(3)+2\left(C_{12}(3)+C_{2}(5)\right)+C_{22}(3)\right)\right) k_{2} \cdot p_{j}\right)+B_{0}\left(i_{4}\right)\left(k_{1}+k_{2}\right) \cdot p_{j}\left(m _ { j } \left(\left(m_{j}^{2}+m_{k}^{2}-m_{H}^{2}\right) \omega_{k j}^{*}\right.\right. \\
& \left.\left.\left.\left.+2 m_{j} m_{k} \omega_{k j}\right)+2\left(m_{j} \omega_{k j}^{*}+2 m_{k} \omega_{k j}\right)\left(k_{1} \cdot k_{2}+\left(k_{1}+k_{2}\right) \cdot p_{j}\right)\right)\right]\right\}, \\
& F_{11_{R}}=\frac{\omega_{k j}^{*} \omega_{i k} m_{W}^{2}}{4 k_{1} \cdot p_{j}}\left\{2\left(B_{0}\left(i_{4}\right)-B_{0}\left(i_{2}\right)-m_{H}^{2} C_{0}(3)+m_{k}^{2} C_{0}(3)-m_{j}^{2}\left(C_{0}(3)+2\left(C_{1}(5)+C_{2}(5)\right)\right)-2 C_{2}(5) k_{1} \cdot k_{2}\right)\right. \\
& -4\left(C_{0}(3)+C_{1}(5)+C_{1}\left(i_{0}\right)+C_{2}(5)-2 D_{00}(1)+2 D_{1}(1) k_{1} \cdot k_{2}\right) k_{1} \cdot p_{j}-4 C_{2}(5) k_{2} \cdot p_{j} \\
& \left.+\frac{1}{k_{1} \cdot k_{2}+\left(k_{1}+k_{2}\right) \cdot p_{j}}\left[m_{H}^{2} B_{0}\left(i_{0}\right)-\left(m_{H}^{2}-m_{k}^{2}\right)\left(B_{0}\left(i_{1}\right)-1\right)-m_{k}^{2} B_{0}\left(i_{2}\right)\right]\right\}, \\
& F_{13_{R}}=\frac{m_{W}^{2}}{4}\left\{4 \omega_{k j}^{*} \omega_{i k}\left(C_{1}\left(i_{0}\right)-C_{2}(1)-2 D_{00}(1)\right)-\frac{\omega_{k j}^{*} \omega_{i k} B_{0}\left(i_{4}\right)}{k_{1} \cdot p_{j}}\left[1-\frac{m_{k}^{2}-m_{H}^{2}}{m_{j}^{2}+2\left(k_{1} \cdot k_{2}+\left(k_{1}+k_{2}\right) \cdot p_{j}\right)}\right]\right. \\
& +\frac{1}{k_{1} \cdot p_{j}}\left[\frac { 1 } { m _ { j } } \left[m_{j} \omega_{k j}^{*} \omega_{i k}\left(B_{0}(1)+\frac{2\left(m_{H}^{2}-m_{k}^{2}\right)}{m_{j}^{2}-m_{i}^{2}}\right)+\frac{2 m_{H}^{2} m_{j} \omega_{k j}^{*} \omega_{i k} B_{0}\left(i_{0}\right)}{m_{j}^{2}-m_{i}^{2}}+\frac{1}{m_{j}^{2}-m_{i}^{2}}\left(\left(\left(m_{k}^{2}-m_{H}^{2}\right)\right.\right.\right.\right. \\
& \left.\times\left(m_{j} \omega_{k j}^{*} \omega_{i k}-m_{i} \omega_{i k}^{*} \omega_{k j}\right) B_{0}\left(i_{1}\right)\right)-2 m_{j}\left(m_{j}^{2}-m_{i}^{2}+m_{k}^{2}\right) \omega_{k j}^{*} \omega_{i k} B_{0}\left(i_{2}\right)+\left(m _ { j } \omega _ { k j } ^ { * } \left(2 m_{i} m_{k} \omega_{i k}^{*}\right.\right. \\
& \left.\left.\left.+\left(m_{j}^{2}+m_{k}^{2}-m_{H}^{2}\right) \omega_{i k}\right)+\left(m_{i}\left(m_{j}^{2}+m_{k}^{2}\right) \omega_{i k}^{*}-m_{H}^{2} m_{i} \omega_{i k}^{*}+2 m_{j}^{2} m_{k} \omega_{i k}\right) \omega_{k j}\right) B_{0}\left(i_{3}\right)\right)+2 m_{j} \\
& \times\left(m_{i} \omega_{i k}^{*}\left(m_{k} \omega_{k j}^{*}+m_{j} \omega_{k j}\right)+\omega_{i k}\left(m_{k}\left(m_{k} \omega_{k j}^{*}+m_{j} \omega_{k j}\right)-m_{H}^{2} \omega_{k j}^{*}\right)\right) C_{0}(1)+4 m_{j} \omega_{k j}^{*} \omega_{i k} C_{00}(1) \\
& +4 m_{j} \omega_{k j}^{*} \omega_{i k} C_{00}(3)-2 m_{j}^{2}\left(m_{j} \omega_{k j}^{*} \omega_{i k}-m_{i} \omega_{i k}^{*} \omega_{k j}\right) C_{1}(1)+2 m_{j} m_{i}\left(m_{j} \omega_{i k}^{*} \omega_{k j}-\left(m_{i} \omega_{k j}^{*} \omega_{i k}\right)\right) C_{2}(1) \\
& +\frac{1}{m_{i}\left(m_{i}^{2}-m_{j}^{2}-2 k_{1} \cdot p_{j}\right)}\left(m _ { j } \left(m_{i} \omega_{k j}^{*}\left(\left(m_{H}^{2}-m_{i}^{2}-m_{k}^{2}\right) \omega_{i k}-2 m_{i} m_{k} \omega_{i k}^{*}\right)-m_{j}\left(\left(m_{i}^{2}+m_{k}^{2}\right) \omega_{i k}^{*}\right.\right.\right. \\
& \left.\left.-m_{H}^{2} \omega_{i k}^{*}+2 m_{i} m_{k} \omega_{i k}\right) \omega_{k j}\right) B_{0}(1)-\left(m_{j}^{2}-m_{i}^{2}\right)\left(m_{H}^{2}-m_{k}^{2}\right) \omega_{i k}^{*} \omega_{k j} B_{0}\left(i_{1}\right)+m_{i}\left(2 m_{j}\left(m_{i}^{2}-m_{j}^{2}\right)\right. \\
& \times \omega_{k j}^{*} \omega_{i k} B_{0}\left(i_{2}\right)+\left(m_{j} \omega_{k j}^{*}\left(2 m_{i} m_{k} \omega_{i k}^{*}+\left(m_{j}^{2}+m_{k}^{2}-m_{H}^{2}\right) \omega_{i k}\right)+\left(m_{i}\left(m_{j}^{2}+m_{k}^{2}\right) \omega_{i k}^{*}-m_{H}^{2} m_{i} \omega_{i k}^{*}\right.\right. \\
& \left.\left.+2 m_{j}^{2} m_{k} \omega_{i k}\right) \omega_{k j}\right) B_{0}\left(i_{3}\right)-2 m_{j}\left(m_{j}^{2}-m_{i}^{2}\right)\left(\omega _ { i k } \left(m_{H}^{2} \omega_{k j}^{*} C_{0}(1)-m_{k}^{2} \omega_{k j}^{*} C_{0}(1)-m_{j} m_{k} \omega_{k j} C_{0}(1)\right.\right. \\
& \left.-2 \omega_{k j}^{*} C_{00}(1)+m_{j}^{2} \omega_{k j}^{*} C_{1}(1)\right)+m_{i}^{2} \omega_{k j}^{*} \omega_{i k} C_{2}(1)-m_{i} \omega_{j k}^{*}\left(m_{k} \omega_{k j}^{*} C_{0}(1)+m_{j} \omega_{k j}\left(C_{0}(1)+C_{1}(1)\right.\right. \\
& \left.\left.\left.\left.\left.\left.\left.+C_{2}(1)\right)\right)\right)\right)+\frac{m_{j}^{2}\left(m_{H}^{2}-m_{k}^{2}\right) \omega_{j k}^{*} \omega_{k j}\left(B_{0}(1)-B_{0}\left(i_{1}\right)\right)}{m_{i}\left(m_{j}^{2}+2 k_{1} \cdot p_{j}\right)}\right]+\frac{\left(m_{H}^{2}-m_{k}^{2}\right) \omega_{k j}^{*} \omega_{j k} B_{0}\left(i_{1}\right)}{m_{j}^{2}+2\left(k_{1} \cdot k_{2}+\left(k_{1}+k_{2}\right) \cdot p_{j}\right)}\right]\right\}, \\
& F_{15_{R}}=-2 \omega_{k j}^{*} \omega_{j k} m_{W}^{4}\left(D_{12}(1)+D_{23}(1)\right) \text {, } \\
& F_{17_{R}}=-\frac{\omega_{k j}^{*} \omega_{j k} m_{W}^{4}\left(C_{12}(3)+C_{2}(5)+C_{22}(3)-2 D_{12}(1) k_{1} \cdot p_{j}\right)}{k_{1} \cdot p_{j}}, \\
& F_{19_{R}}=\frac{\omega_{j k} m_{W}^{3}}{8 k_{1} \cdot p_{j}}\left\{4\left(m_{k} \omega_{k j} C_{0}(3)+m_{j} \omega_{k j}^{*}\left(C_{0}(3)+C_{1}(5)+C_{2}(5)\right)\right)+8\left(m_{k} \omega_{k j} D_{0}(1)-m_{j} \omega_{k j}^{*} D_{2}(1)\right) k_{1} \cdot p_{j}\right. \\
& +\frac{k_{1} \cdot p_{j} k_{2} \cdot p_{j}}{g\left(k_{1}, k_{2}\right)}\left[m_{j}\left(m_{H}^{2}-m_{k}^{2}\right) \omega_{k j}^{*} B_{0}\left(i_{1}\right)+B_{0}\left(i_{4}\right)\left(m_{j}\left(\left(m_{j}^{2}+m_{k}^{2}-m_{H}^{2}\right) \omega_{k j}^{*}+2 m_{j} m_{k} \omega_{k j}\right)\right.\right. \\
& \left.\left.\left.+2\left(m_{j} \omega_{k j}^{*}+2 m_{k} \omega_{k j}\right)\left(k_{1} \cdot k_{2}+\left(k_{1}+k_{2}\right) \cdot p_{j}\right)\right)\right]\right\} \text {, } \\
& F_{21}=\frac{8 m_{W}^{2}}{2 k_{1} \cdot k_{2}}\left(3+\frac{2 k_{1} \cdot k_{2}}{2 m_{W}^{2}}+6 m_{W}^{2}\left(1-\frac{2 k_{1} \cdot k_{2}}{2 m_{W}^{2}}\right) C_{0}(i)\right)-Q_{t}^{2} N_{c_{t}} \frac{8 m_{t}^{2}}{2 k_{1} \cdot k_{2}}\left(2+\left(4 m_{t}^{2}-2 k_{1} \cdot k_{2}\right) C_{0}(I I)\right) \text {. }
\end{aligned}
$$


[1] For a recent review, see M. Battaglia, arXiv:0705.3997 1 [hep-ph].

[2] Flavor changing neutral currents transitions of the quark top within the standard model have been studied by diverses authors. See for instance, J. L. Díaz-Cruz, R. Martínez, M. A. Pérez, and A. Rosado, Phys. Rev. D41, 891 (1991); G. Eilam, J. L. Hewett, and A. Soni, Phys. Rev. D44, 1473 (1991); 59, 039901 (E) (1999); N. G. Deshpande, B. Margolis, and H. D. Trottier, Phys. Rev. D45, 178 (1992); B. Mele and S. Petrarca, Phys. Lett. B435, 401 (1999); A. Cordero-Cid, J. M. Hernández, G. Tavares-Velasco, and J. J. Toscano, Phys. Rev. D73, 094005 (2006).

[3] G. Eilam, M. Frank, and I. Turan, Phys. Rev. D73, 053011 (2006); G. Eilam, M. Frank and I. Turan, Phys. Rev. D74, $035012(2006)$.

[4] W. Buchmuller and D. Wyler, Nucl. Phys. B286, 621 (1986); J. Wudka, Int. J. Mod. Phys. A9, 2301 (1994).

[5] Adriana Cordero-Cid, M. A. Pérez, G. Tavares-Velasco, and J. J. Toscano, Phys. Rev. D70, 074003 (2004); J. L. DíazCruz and J. J. Toscano, Phys. Rev. D62, 116005 (2000); F. del Aguila, M. Pérez-Victoria, and J. Santiago, Phys. Lett. B492, 98 (2000).

[6] J. I. Aranda, F. Ramírez-Zavaleta, J. J. Toscano, and E. S. Tututi, Phys. Rev. D78, 017302 (2008) (arXiv:0804.2652).

[7] See for instance, J. L. Díaz-Cruz, R. Noriega-Papaqui, and A. Rosado, Phys. Rev. D69, 095002 (2004); Phys. Rev. D71, $015014(2005)$.

[8] J. L. Feng, T. Moroi, H. Murayam, and E. Schnapka, Phys. Rev. D57, 5875 (1998).

[9] I. Dorsner and S. M. Barr, Phys. Rev. D65, 095004 (2002).

[10] J. I. Aranda, A. Flores-Tlalpa, F. Ramírez-Zavaleta, F. J. Tlachino, J. J. Toscano, and E. S. Tututi, in preparation.

[11] G. Passarino and M. J. G. Veltman, Nucl. Phys. B160, 151 (1979).

[12] R. Mertig, M. Bohm, and A. Denner, Comput. Phys. Commun. 64, 345 (1991).

[13] See for instance, J. F. Gunion, H. E. Haber, G. Kane, and S. Dawson, The Higgs Hunters Guide, 2nd ed., Frontiers in Physics Vol. 80 (Addision-Wesley, Reading MA, 1990).

[14] C. Pagliarone, A. Fernandez and J. J. Toscano, Frascati Physics Series Vol. XLVI (2007), arXiv:0802.1949 [hep-ph]; A. Fernández, C. Pagliarone, F. Ramírez-Zavaleta, and J. J. Toscano, submitted to Phys. Rev. D.

[15] C. Amsler et al. [Particle Data Group], Phys. Lett. B667, 1 (2008).

[16] ALEPH Collab., Phys. Lett. B526, 191 (2002); DELPHI Collab. Eur. Phys. J. C32, 145 (2004); L3 Collab., Phys. Lett. B517, 319 (2001); OPAL Collab., Eur. Phys. J. C26, 479 (2003); ALEPH, DELPHI, L3, OPAL, The LEP Working Group for Higgs Boson Search, Phys. Lett. B565, 61(2003).

[17] V. Telnov, Nucl. Instr. Meth. A294, 72 (1990). 